\title{
RIEMANN PROBLEMS FOR NONSTRICTLY HYPERBOLIC $2 \times 2$ SYSTEMS OF CONSERVATION LAWS
}

\author{
DAVID G. SCHAEFFER AND MICHAEL SHEARER
}

\begin{abstract}
The Riemann problem is solved for $2 \times 2$ systems of hyperbolic conservation laws having quadratic flux functions. Equations with quadratic flux functions arise from neglecting higher order nonlinear terms in hyperbolic systems that fail to be strictly hyperbolic everywhere. Such equations divide into four classes, three of which are considered in this paper. The solution of the Riemann problem is complicated, with new types of shock waves, and new singularities in the dependence of the solution on the initial data. Several ideas are introduced to help organize and clarify the new phenomena.
\end{abstract}

1. Introduction. The Riemann problem for a $2 \times 2$ system of conservation laws in one space variable $x$ and time $t$ is the initial value problem

$$
\begin{gathered}
U_{t}+F(U)_{x}=0, \quad-\infty<x<\infty, t>0, \\
U(x, 0)=\left[\begin{array}{ll}
U_{L} & \text { if } x<0, \\
U_{R} & \text { if } x>0 .
\end{array}\right.
\end{gathered}
$$

Here $U=U(x, t) \in \mathbf{R}^{2}$, while $U_{L}, U_{R}$ in $\mathbf{R}^{2}$ are given constants. We suppose that (1.1) is hyperbolic so that the eigenvalues $\lambda_{k}(U)(k=1,2)$ of $d F(U)$ are real. Our purpose is to study the Riemann problem near an isolated umbilic point; i.e., a point $U$ when $\lambda_{1}(U)=\lambda_{2}(U)$ and $d F(U)$ is diagonalizable. (At such a point $d F(U)$ is a multiple of the identity.) It was shown in [13] that by means of a linear change of dependent variable, a $2 \times 2$ hyperbolic system with an isolated umbilic point could be reduced near the umbilic point, modulo higher order terms, to the normal form

$$
U_{t}+d C(U)_{x}=0
$$

where

$$
C(u, v)=a u^{3} / 3+b u^{2} v+u v^{2} .
$$

The behavior of solutions of (1.3) depends on the parameters $a$ and $b$ in a subtle way. In [13] four qualitatively different cases were identified, corresponding to a partition of the $(a, b)$-plane into four regions. In this paper we solve the Riemann problem for (1.3) in three of the four cases (Cases II-IV of [13], see Figure 1). (The

Received by the editors August 8, 1986 and, in revised form, December 1, 1986.

1980 Mathematics Subject Classification (1985 Revision). Primary 35L65, 76T05.

The first author was supported in part by NSF Grant MCS-8401591.

The second author was supported in part by U. S. Army Research Grant DAAL03-86-K0004, FINEP/Brazil Grant 4.3.82.017.9, CNPq/Brazil Grant 1.01.10.011/84-ACE and NSF Grant No. 5274298 INT-8415209. 


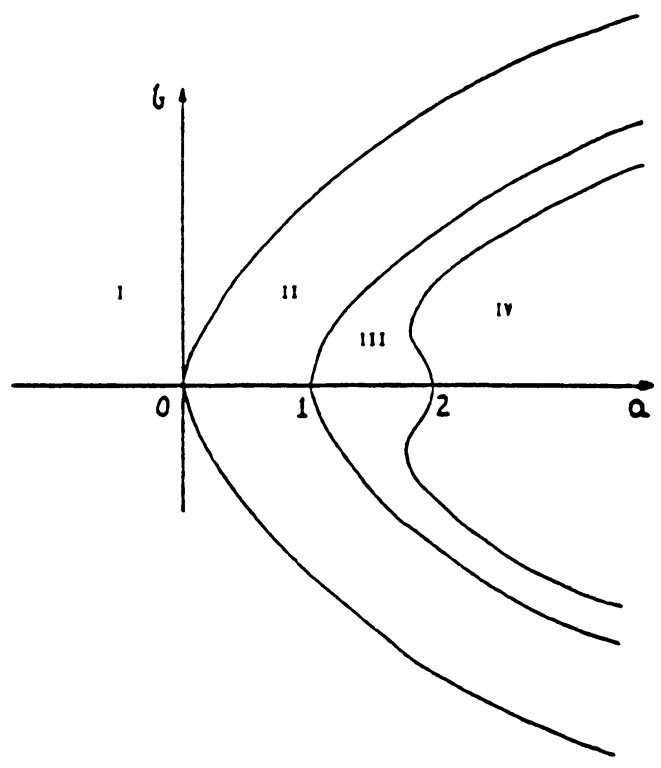

FIGURE 1. The four cases

fourth case, which involves the new issue of undercompressive shocks, is not discussed here; however, cf. [14] for the solution in a special case, and [15] for the solution when $b=0$ and both $U_{L}$ and $U_{R}$ are restricted to the upper half plane.)

The present study was motivated in part by the problem of tracking fronts in a two-dimensional oil reservoir. When a multiphase flow near the front is approximated locally by a one-dimensional flow, the front can be tracked by solving a Riemann problem [3, 4]. For certain models of three-phase flow, equations (1.1) are a hyperbolic system with isolated umbilic points [13]. (For other models, the equations are of mixed hyperbolic/elliptic type [1]. The Riemann problem for a related system of mixed type is solved in [6].)

The Riemann problem for (1.3) exhibits several new phenomena not occurring for strictly hyperbolic equations. A minor example is that for certain ranges of data, the solution of the $2 \times 2$ system (1.3) may consist of three distinct waves, separated in the $(x, t)$-plane by constant states. However, the main new phenomena arise from overcompressive waves, especially overcompressive shocks. An overcompressive shock (for a $2 \times 2$ system) is a shock such that both characteristics on both sides of the shock enter the shock. (In the $n \times n$ case, a total of $n+2$ characteristics enters an overcompressive shock.) It turns out that many wave curves in the $U_{R}$-plane end at a point representing an overcompressive shock, and they cannot be continued. (In the strictly hyperbolic case [11], wave curves continue indefinitely.) Because the wave curves terminate, the solution of the Riemann problem, considered as a function of $U_{R}$, has certain jump discontinuities. Overcompressive shocks also raise some challenging questions regarding the choice of entropy condition. For our problem, we get existence and uniqueness using the Lax criterion [10]; in order to obtain 
existence, we specifically need certain shocks which violate condition (E) of [11]. On the other hand, it appear that for more complicated models of three-phase flow, the Lax condition will not be sufficient to obtain uniqueness; rather, one will need a generalization of condition (E), to include shocks parametrized by a detached wave curve in $U_{R}$-space; i.e., a curve not connected to $U_{L}$ (cf. $\S 5$ ).

Unfortunately, these new phenomena may be obscured by the complexity of the solution of the Riemann problem for (1.3). As a reference point, consider the Riemann problem (in the small) for a $2 \times 2$ strictly hyperbolic, genuinely nonlinear system: for fixed $U_{L}$, the $U_{R}$-plane divides into four regions, corresponding to solutions containing a 1-shock and a 2-shock, a 1-rarefaction and a 2-shock, etc. By contrast, for fixed $U_{L}$, the solution of the Riemann problem for (1.3) may divide the $U_{R}$-plane into as many as fifteen regions, representing different combinations of shocks, rarefactions, and composite waves. The boundaries of these regions are significant because the solution of the Riemann problem, considered as a function of $U_{R}$, is singular across them; these singularities are often much stronger than the mild singularities which occur in the strictly hyperbolic case. Further complicating the picture, the topology of the $U_{R}$-regions may vary as $U_{L}$ varies.

Because of these technical complications, we have put a lot of effort into the exposition of this paper in an attempt to make the information accessible to the reader. In particular, we have included:

(i) Two different representations of the solution of the Riemann problem and

(ii) A phenomenological catalogue of boundary curves in both the $U_{R^{-}}$and $U_{L}$-planes.

Regarding (i): The first representation, which shows the coordinate system arising from the slow wave-fast wave construction, exhibits the gross qualitative features of the solution. The second representation, which shows the precise combination of shock, rarefaction, and composite waves in the solution, gives a more complete description. Regarding (ii): By $U_{R}$-boundary curves we mean the boundaries of the $U_{R}$-regions discussed above, and by $U_{L}$-boundary curves we mean curves in the $U_{L}$-plane across which the qualitative structure of the $U_{R}$-regions changes. We anticipate that the catalogue of $U_{R}$-boundaries will prove helpful in understanding the new phenomena.

In the main text of this paper, we restrict ourselves to solving the Riemann problem for (1.3) in one of the four cases of [13] (see Figure 1); specifically Case II, defined by the inequality $\frac{3}{4} b^{2}<a<1+b^{2}$. Essentially all of the complications in Cases II-IV arise in Case II. (In Appendix 2, written jointly with E. Isaacson and D. Marchesin, we present the solutions for Cases III and IV.) Our main results are stated in $\$ \S 2$ and 3. Specifically, $\$ 2$ studies the wave curves of each characteristic family separately; by applying bifurcation theory in studying the shock curves, we are able to achieve some completeness in classifying the $U_{L}$-boundaries. Then $\S 3$ synthesizes this information into the solution of the Riemann problem; in particular, $\$ 3$ contains the two representations of the solution and the catalogue of boundaries mentioned above. In $\S 4$ we prove several lemmas stated in $\$ 2$ concerning $U_{L^{-}}$ boundaries. In $\$ 5$ we analyze what composite waves are possible for (1.3); this analysis leads naturally to a discussion of shock admissibility conditions for (1.3). 
Appendix 1 catalogues intersection points in the $U_{R}$-plane (i.e., points where two boundary curves cross), and as mentioned above, Appendix 2 solves the Riemann problem in Cases III and IV.

The results of this paper were obtained through a combination of numerical and analytical techniques involving several collaborators. First the Hugoniot loci were obtained numerically in the symmetric case (i.e., $b=0$ ), using a computer program written by E. Isaacson, D. Marchesin and B. Plohr. This information was combined with the rarefaction curves, given analytically in [13] and numerically by the computer program, to deduce the solution of the Riemann problem in [7-9], in Cases II-IV with $b=0$. To study $b \neq 0$, ideas from bifurcation theory, introduced in [14], were used to show that breaking the symmetry led to a hysteresis phenomenon that does not occur in the symmetric cases. The Hugoniot loci were then found numerically for representative nonsymmetric cases, together with the location of the $U_{L}$ boundary curve corresponding to the hysteresis. This information is combined with analytical considerations in the present paper. We are grateful to the authors of [7-9] for making rough drafts of their papers available to us at an early stage. Incidentally, Cases II-IV were solved in the opposite order from that presented here; specifically, Case IV, the simplest technically, was solved first, through the joint efforts of the four authors of Appendix II.

Because of the dependence upon computations, the solutions of Riemann problems given here and in [7-9] are not fully substantiated by rigorous proofs. To see the significance of this point, recall that the reduction to the normal form (1.3) is only modulo higher order terms. One would like to show that the inclusion of higher order terms does not change the qualitative structure of solutions of the Riemann problem. Some results concerning invariance under such perturbations are given in [13]. However, to understand the full effect of perturbations, a rigorous analysis of the Riemann problem for (1.3) seems to be needed. This paper contains two contributions towards this goal. First, we have proved (in \$4) various statements made about $U_{L}$-boundaries, and second, the catalogue of $U_{R}$-boundaries (in $\S 3(\mathrm{c})$ ), which includes defining equations, helps establish a possible framework for a rigorous analysis of the $U_{R^{-}}$-boundaries. This work extends the study of $U_{L^{-}}$and $U_{R}$-boundaries as appears in $[7,14]$. Current work of E. Isaacson, D. Marchesin and B. Plohr on these boundaries seeks a more general classification of boundaries than that presented here, in which the restriction of quadratic nonlinearities is removed. We are grateful to B. Plohr for sharing his preliminary notes on this subject.

2. Wave curves for Case II. In this section we discuss the wave curves of (1.3), representing rarefactions, shocks, or composite waves in a single characteristic family, from which solutions of the Riemann problem in Case II will be constructed. For strictly hyperbolic equations for which genuine nonlinearity may fail, Liu [11] has solved the Riemann problem using composite waves to continue the wave curves beyond weak waves. We draw on his work in constructing wave curves for (1.3), but since strict hyperbolicity fails for (1.3), these curves exhibit new behavior; specifically there are detached wave curves (in other words, the wave curve may have more than one component) and in some cases a wave curve stops at some finite amplitude 
rather than continuing indefinitely as in [11]. The study of shock curves occupies most of this section; as in [14], we use bifurcation ideas to understand these curves.

(a) Rarefaction curves and the inflection locus. Rarefaction waves are piecewise smooth functions $U(x / t)$ that satisfy (1.3). The values of $U(\xi)$ must lie on an integral curve of one of the right eigenvectors $r_{k}(U)\left(k=1\right.$ or 2) of $d^{2} C(U)$, while $\xi=x / t$ is the corresponding characteristic speed $\lambda_{k}(U(\xi))$. Thus $\lambda_{k}(U(\xi))$ must increase monotonically with $\xi$. This requirement gives a direction (namely that of increasing characteristic speed) to the integral curves of $r_{k}(U)$. The oriented integral curves are called rarefaction curves. The patterns of these curves (cf. [13]) in Cases I-IV are shown in Figure 2. Note that there are points at which $\lambda_{k}(U)$, when restricted to the integral curve, has a critical point. Such a point $U$ is called an inflection point, and satisfies

$$
d \lambda_{k}(U) \cdot r_{k}(U)=0 \text {. }
$$

Equation (2.1) corresponds to a loss of genuine nonlinearity at $U$. Solutions of equation (2.1) typically form curves in state space, called inflection loci. The term inflection is motivated by the situation for a single conservation law $u_{t}+f(u)_{x}=0$, for which the characteristic speed $\lambda(u)=f^{\prime}(u)$ has a critical point at an inflection point of $f$.

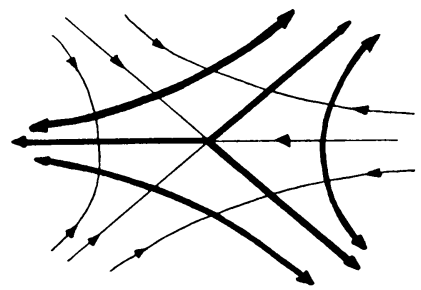

Case I

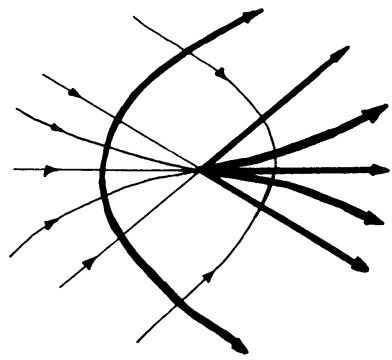

Case III

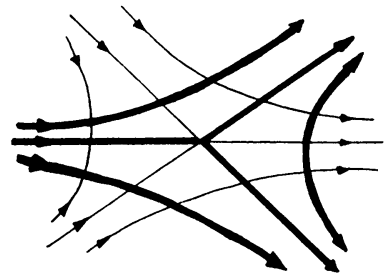

Case II

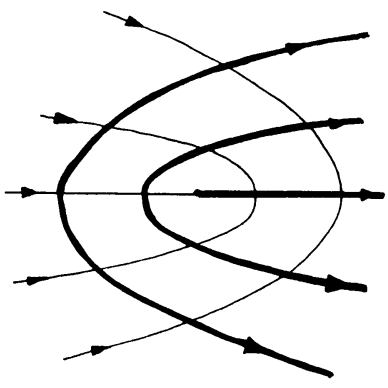

Case IV

$\longrightarrow \quad$ slow waves
$\rightarrow \quad$ fast waves

FIGURE 2. Schaeffer/Shearer: Riemann problems 


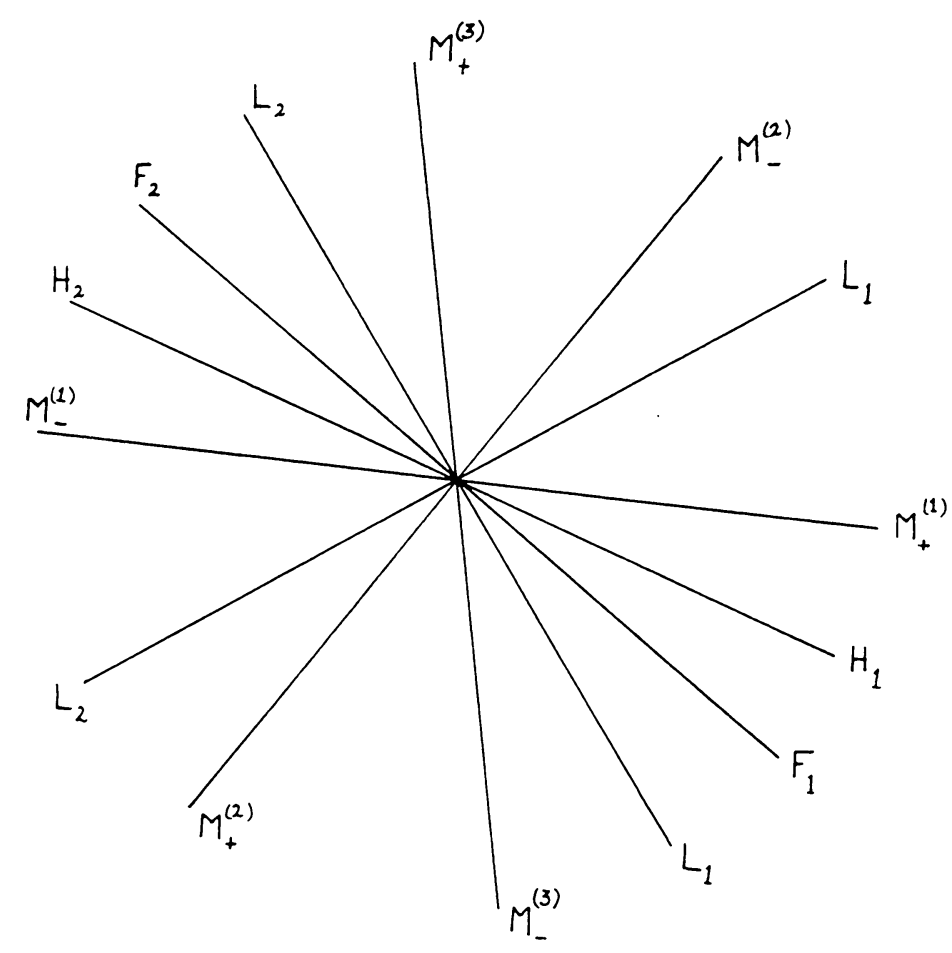

Figure 3. $U_{I}$, sectors, Case II

(b) Shock curves and boundaries in the $U_{I}$-plane. If we consider shock waves that are jump discontinuities between constant values $U_{L}$ and $U$ of $U(x, t)$, then the shock speed is a constant. The triple $\left(s, U_{L}, U\right)$ must satisfy the Rankine-Hugoniot condition

$$
G\left(s, U, U_{L}\right) \equiv-s\left(U-U_{L}\right)+Q(U)-Q\left(U_{L}\right)=0
$$

in order that the jump discontinuity be part of a weak solution of equation (1.3). (We have written $Q(U)$ in place of $d C(U)$.)

For fixed $U_{L}$, equation (2.2) may be regarded as a bifurcation problem for $(s, U)$. The trivial solution $U=U_{I}$ represents the constant solution $U(x, t)=U_{I}$ of equation (1.3). We shall call the solution set $\{(s, U): G=0\}$ of (2.2) a bifurcation diagram.

As $U_{L}$. varies, the bifurcation diagram changes also. However, for most $U_{L}$, the essential qualitative structure of the diagram is unchanged by small perturbations of $U_{L}$, even though the precise shape of the bifurcation diagram does change. Specifically for Case II, in Figure 3 we identify sectors in the $U_{L}$-plane with the property that for all $U_{I}$ in any one sector, the bifurcation diagram is qualitatively the same (in a sense to be clarified below). The bifurcation diagrams are shown in Figure 4; Figure 5 contains the corresponding pictures of the Hugoniot loci, which are the projections of the bifurcation diagrams onto the $U_{R}$-plane. (Similar diagrams for Cases III and IV are given in Figures 14, 15, 19, 20 of Appendix 2.) Figures 3, 4 and 


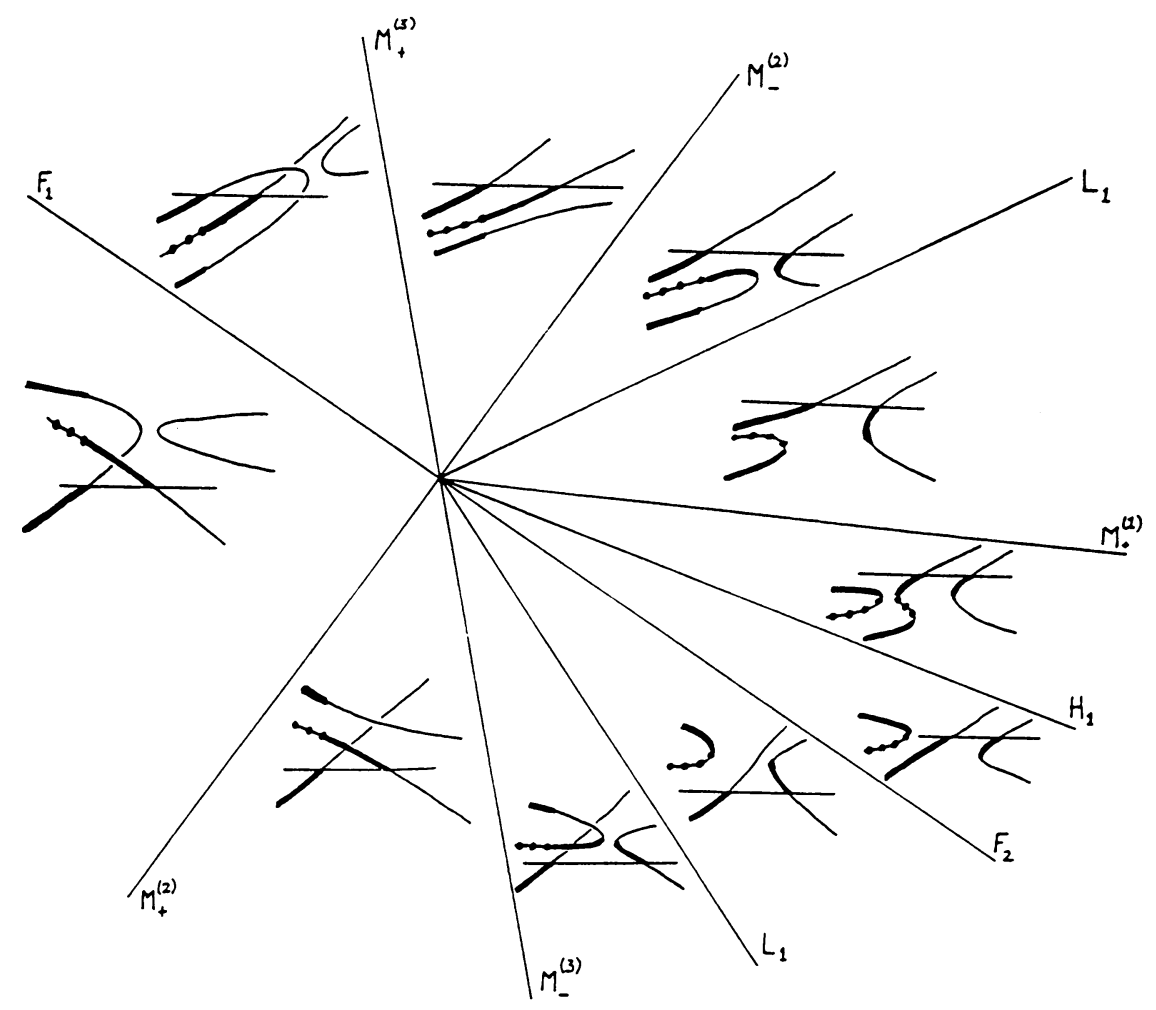

Graphs of $U_{R}$ (vertical axis) vs. $s$ (horizontal axis)

FIgURE 4. Bifurcation diagrams, Case II

5 are for generic $(a, b)$ in Case II, in particular with $b \neq 0$. (If $b$ were equal to zero, Figure 3 would be distorted as follows. The figure would be symmetric about the median $M^{(1)}$, and some of the boundary rays would coalesce, thereby reducing the number of sectors, cf. [9].)

It is not important at this stage to fully understand all the detail in Figures 4 and 5. We shall focus on the changes in the qualitative structure of the bifurcation diagrams across sector boundaries, as these relate to the Lax admissibility criterion for shocks. If $s$ is the speed of a shock in a weak solution

$$
U(x, t)=\left[\begin{array}{ll}
U_{L} & \text { for } x<s t \\
U_{R} & \text { for } x>s t
\end{array}\right.
$$

then $U(x, t)$ is an admissible 1-shock if

$$
s<\lambda_{1}\left(U_{l}\right), \quad \lambda_{1}\left(U_{R}\right)<s<\lambda_{2}\left(U_{R}\right),
$$

and an admissible 2-shock if

$$
\lambda_{1}\left(U_{L}\right)<s<\lambda_{2}\left(U_{L}\right), \quad s>\lambda_{2}\left(U_{R}\right) .
$$




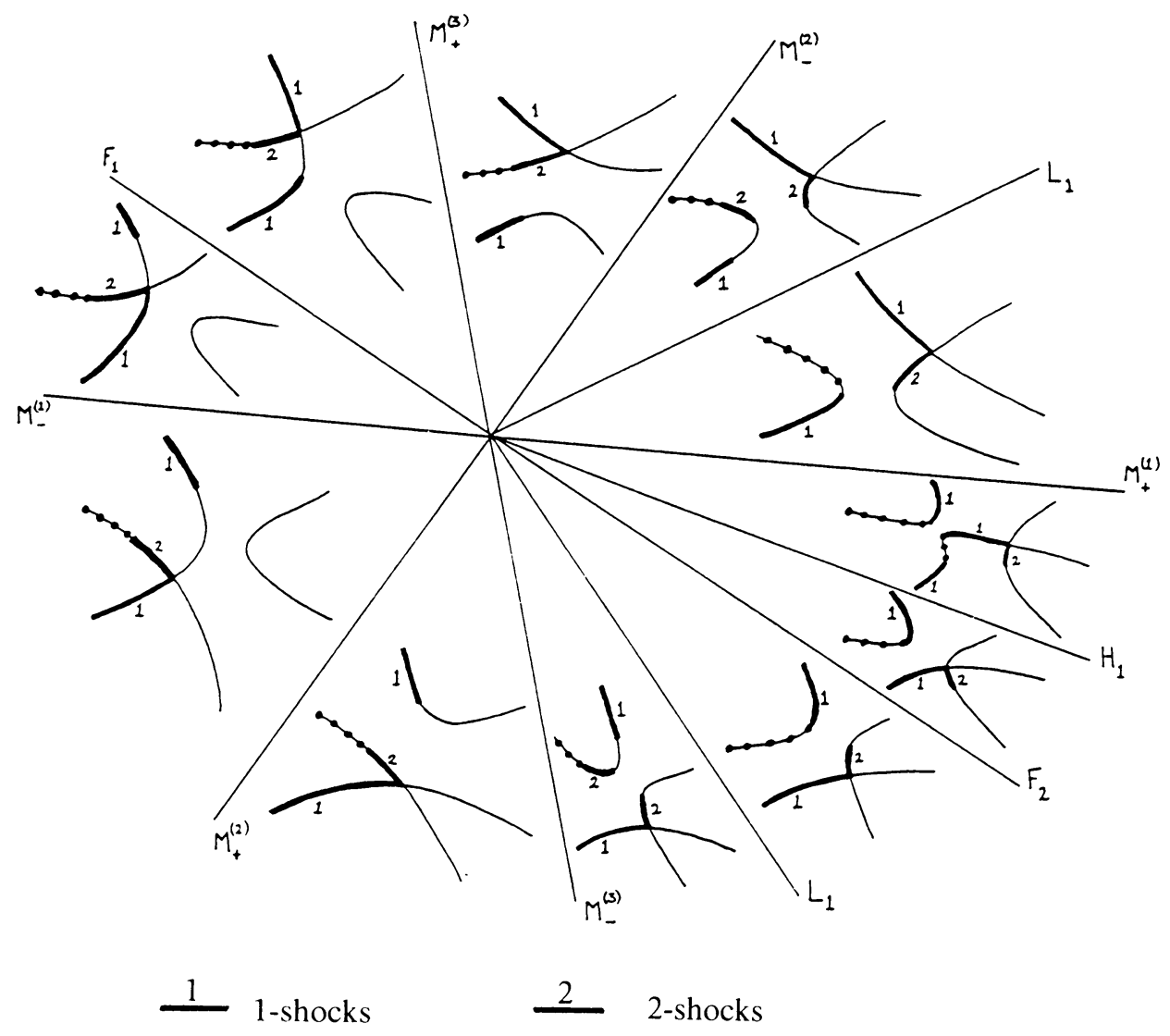

$\longrightarrow$ overcompressive shocks

Figure 5. Hugoniot loci, Case II

In the bifurcation diagrams, since the primary bifurcations occur at $s=\lambda_{k}\left(U_{L}\right)$, $k=1,2$, it may be determined by inspection whether inequalities involving $U_{L}$ are satisfied; specifically $s<\lambda_{1}\left(U_{L}\right)$ if $s$ lies to the left of both primary bifurcation points, and $\lambda_{1}\left(U_{L}\right)<s<\lambda_{2}\left(U_{L}\right)$ if $s$ lies between the primary bifurcation points. As explained in [14], it may be deduced from the bifurcation diagram, using linearized stability ideas, whether inequalities involving $U_{R}$ are satisfied. In particular, the admissibility of a given branch of solutions can change from violation of a $U_{R}$-inequality only at a bifurcation point or a limit point (i.e., a point where $\dot{s}=0$ since the tangent to the curve lies in a plane $s=$ const.). By comparison, the admissibility of a branch can change from violation of a $U_{L}$-inequality only at a primary bifurcation point or an overlap point (i.e.; a point $U \neq U_{L}$, where $s=\lambda_{k}\left(U_{L}\right)$, so called since the bifurcation diagram overlaps the plane $s=\lambda_{k}\left(U_{L}\right)$ of a primary bifurcation). In Figures 4 and 5, the portions of the curves representing admissible shocks are shown in emphasized print. Some of the sectors have been combined in Figures 4, 5 if there are no changes in the diagrams affecting admissible shocks. 

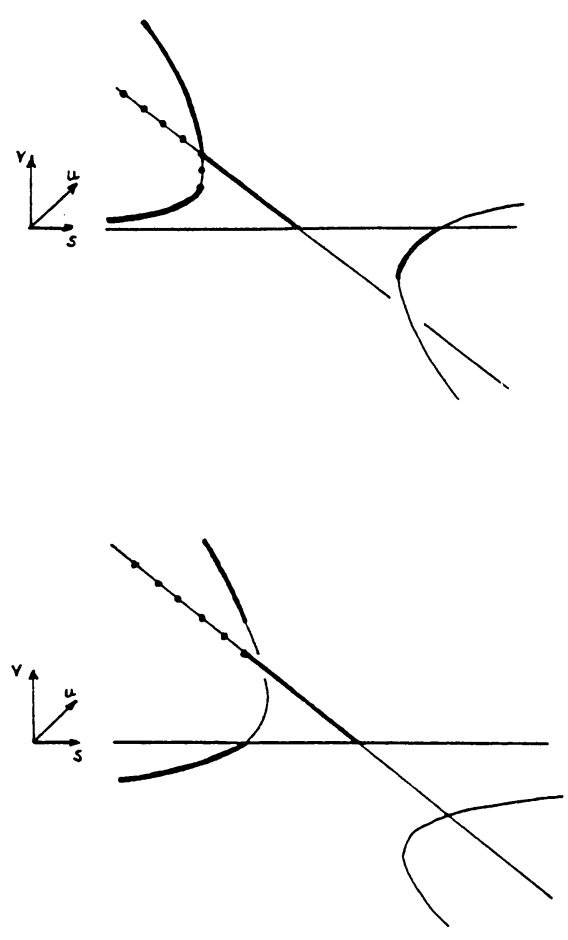

Bifurcation diagrams
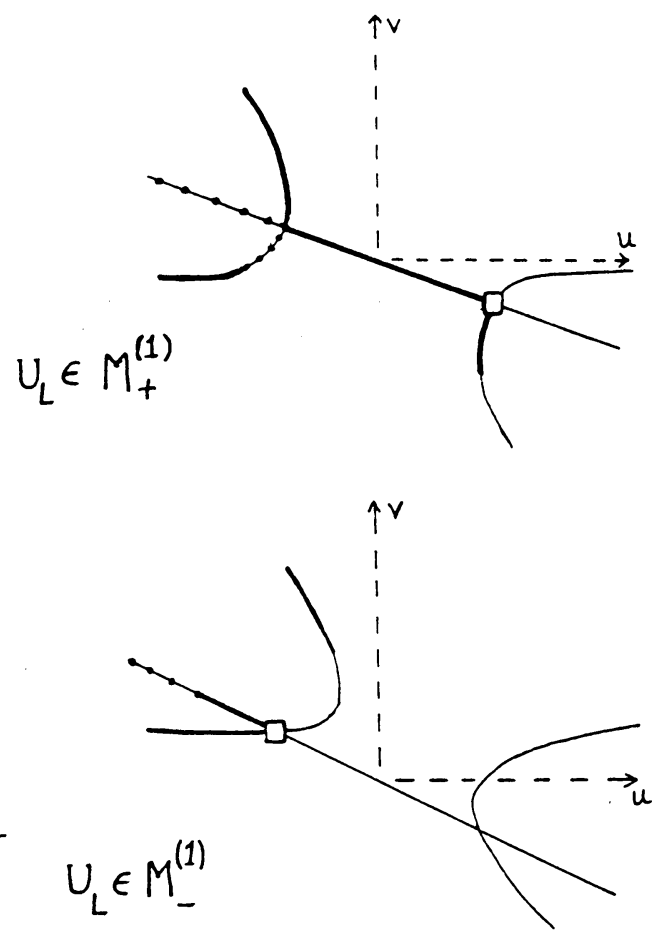

Hugoniot loci

FIGURE 6. $U_{L}$ on median $M^{(1)}$

In Figures 4 and 5, certain other curves are highlighted with dots. These represent overcompressive shocks, for which all the characteristics enter the shock; in symbols

$$
\lambda_{2}\left(U_{R}\right)<s<\lambda_{1}\left(U_{L}\right) \text {. }
$$

Overcompressive shocks, which we discuss in $\S 3$, play an important role in the solution of the Riemann problem.

In order to help the reader understand some of the transitions in Figure 4, we show in Figure 6 bifurcation diagrams and Hugoniot loci for $U_{L}$ on one of the medians $M^{(1)}$. The medians are significant because secondary bifurcation occurs precisely when $U_{L}$ lies on a median (see Lemma 4.2).

We now discuss the distinguished rays in the $U_{L}$-plane that are boundaries of $U_{L}$-sectors in Figure 3. Significant changes occur in the bifurcation diagram across these rays through one of the following four mechanisms:

(i) secondary bifurcation

(ii) limit-overlap

(iii) inflection point or

(iv) hysteresis point.

In Figure 3 these four phenomena are identified by the labels $M$ (for median), $L, F$, and $H$, respectively. The two halves of $L, F$, and $H$ are distinguished by a subscript which indicates which characteristic family is primarily involved; the two halves of 
$M^{(j)}, j=1,2,3$, are distinguished by an arbitrary \pm sign. The relation of the medians and secondary bifurcations was already mentioned above; let us briefly discuss the remaining three phenomena, items (ii)-(iv).

Concerning (ii), limit points and overlap points are ways in which the admissibility of shocks may change, because one of the inequalities of (2.4), (2.5) is violated. For values of $U_{L}$ on $L_{k}(k=1$ or 2$)$, a single point $(s, U)$ is both a limit point and an overlap point. We refer to $(s, U)$ as a limit-overlap. For quadratic nonlinearities, we show in $\S 4$ that $L_{k}$ is the locus of points $U$ satisfying $\lambda_{k}(U)=0, k=1$ or 2 .

Concerning (iii), if $U_{L}$ is an inflection point (i.e. satisfies (2.1)), in symbols $U_{I} \in F_{k}$ ( $k=1$ or 2 ), then the primary bifurcation from $s=\lambda_{k}\left(U_{L}\right)$ is one-sided; i.e. near the bifurcation point the nontrivial solutions lie entirely in a half space $\left\{s>\lambda_{k}\left(U_{L}\right)\right\}$ or $\left\{s<\lambda_{k}\left(U_{L}\right)\right\}$, rather than intersecting both half spaces. Thus at an inflection point $\dot{s}=0$ while $\ddot{s} \neq 0$ on the bifurcation branch at $s=\lambda_{k}\left(U_{L}\right), U=U_{L}$. We prove this property of $F_{k}$ in Lemma 4.1 below.

Finally, concerning (iv), when $U_{I} \in H_{k}$, two limit points coalesce at a point $(s, U)$, giving $\dot{s}=\ddot{s}=0$, while $\ddot{s} \neq 0$. Using the terminology of [5], we call such a point a hysteresis point.

(c) Composite wave curves. In $\$ 5$ we will show that only two sorts of composite waves arise in connection with quadratic nonlinearities; viz., rarefaction-shocks in the first characteristic family and shock-rarefactions in the second. In a 1-rarefaction-shock, the shock speed $s$ equals the fastest characteristic speed $\lambda_{1}$ in the rarefaction so that, in the $(x, t)$-plane, the shock is on the right-hand edge of the rarefaction. Similarly, in a 2-shock-rarefaction, the shock is on the left-hand edge of the rarefaction. Note that the shock in each case is only "marginally" admissible in that one of the inequalities in (2.4) or (2.5) is an equality.

To conclude our discussion of wave curves, for fixed $U_{L}$, we write $W_{k}\left(U_{L}\right), k=1$ or 2, for the set of all $U_{R}$ such that there is a solution of the Riemann problem (1.2), (1.3) involving only $k$-waves. Thus $W_{k}\left(U_{l}\right)$ is a (possibly disconnected) curve in the $U_{R}$-plane which passes through the point $U_{l}$. When no confusion seems likely we may suppress the argument $U_{l}$, writing simply $W_{k}$. When we need more detailed structure concerning wave curves in the $k$-family, we use the notation $R_{k}, S_{k}$, $(R S)_{k}$, or $(S R)_{k}$ for the rarefaction curve, shock curve, rarefaction-shock curve, or shock-rarefaction curve, respectively. We will denote by $S_{0}$ the curve in the $U_{R}$-plane representing overcompressive shocks with $U_{L}$ on the left.

3. Solution of the Riemann problem in Case II. In this section we present solutions of the Riemann problem for (1.3) in Case II. Subsection (a) gives diagrams which show how wave curves representing combinations of successively faster waves starting from $U_{l}$, fill up the $U_{R}$-plane. Subsection (b) explores a more detailed representation of the solution in which the $U_{R}$-plane is divided into regions, each associated with a particular combination of waves needed to solve the Riemann problem. An alternative characterization of these regions results from considering the solution of the Riemann problem as a function of $U_{R}$-this function is piecewise smooth, being $C^{\infty}$ on the interior of each region but singular across the boundaries. (The singularities may involve only a jump in a high order derivative.) Subsection (c) catalogues the phenomena leading to such boundaries. (Appendix 1 further elaborates 
on the boundaries by considering points where two (or more) boundary curves intersect.)

(a) Combinations of wave curves. The Riemann problem is solved by considering all combinations of successively faster waves starting from $U_{L}$. In Figure 8 below, we represent this construction pictorially for several values of $U_{L}$; specifically, we show in bold face print $W_{1}\left(U_{L}\right)$, the 1-wave curve representing all the states $U_{R}$ connected to $U_{L}$ by a physical 1-wave (possibly composite), and we show in lightface print representative 2-wave curves emerging from points on $W_{1}\left(U_{L}\right)$. (The other curves in Figure 8, shown as knotted or as parallel light lines, represent singularities of this coordinate system and will be discussed momentarily.) To solve the Riemann problem, find a point $U^{*}$ on $W_{1}\left(U_{L}\right)$ such that the 2-wave curve $W_{2}\left(U^{*}\right)$ contains $U_{R}$; the solution of the Riemann problem is then a (possibly composite) 1-wave from $U_{L}$ to $U^{*}$, together with a (possibly composite) 2-wave from $U^{*}$ to $U_{R}$. (This algorithm is incomplete in the case of Figure 8c; cf. the discussion of the detached rarefaction curve below.)

Although in principle each $U_{L}$ gives a different system of curves in the $U_{R}$-plane, many of these different systems of curves have the same topological structure. Indeed, their structure changes only when $U_{L}$ crosses one of the rays $M^{(j)}, L, F$, or $H$ in Figure 3; i.e., only when the qualitative structure of the bifurcation diagram of $U_{L}$ changes. Moreover, only a fraction of the rays in Figure 3 actually lead to changes in the topological structure of this system of curves; specifically, only the rays $M_{+}^{(j)}, j=1,2,3$, and $H_{1}$ shown in Figure 7 . These rays divide the $U_{L}$-plane into

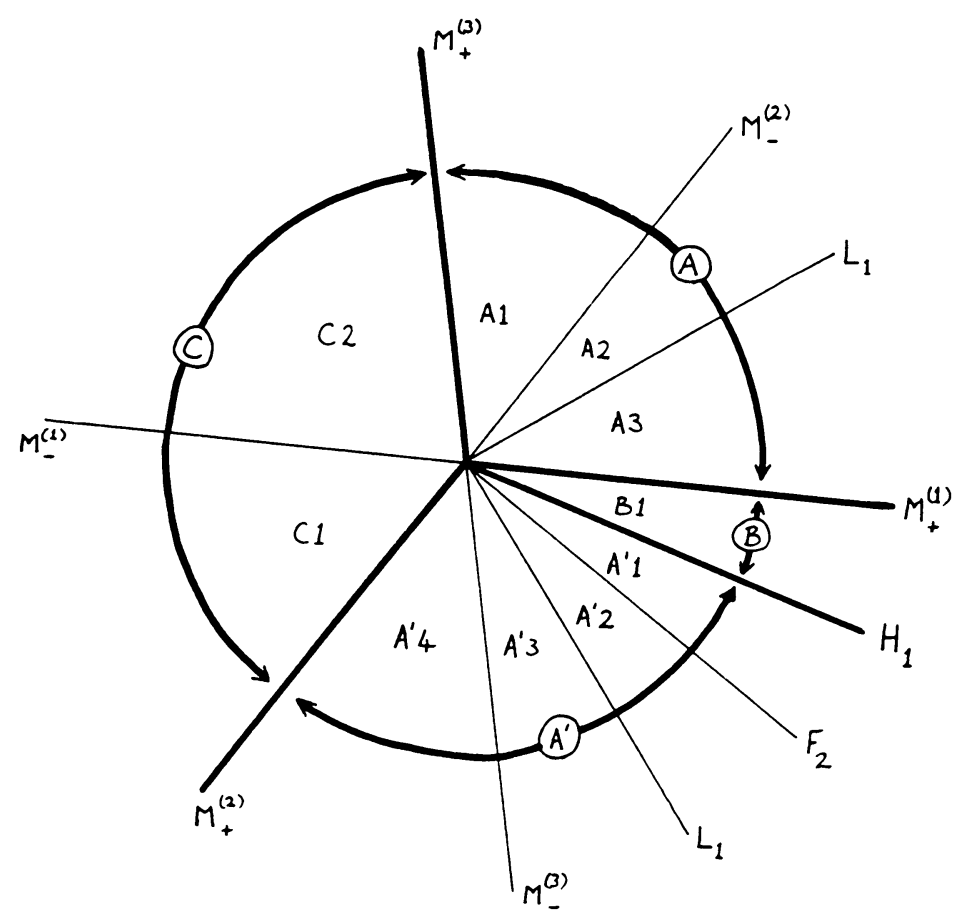

Figure 7. $U_{I}$-boundaries, Case II 


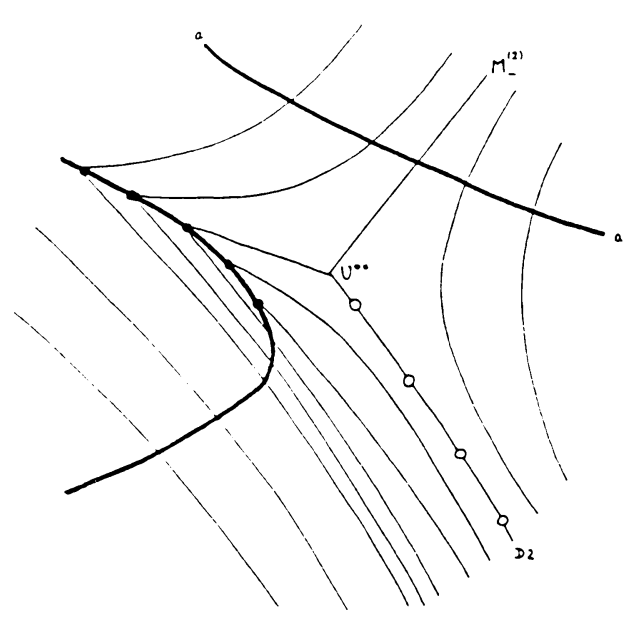

$8 \mathrm{~A}$

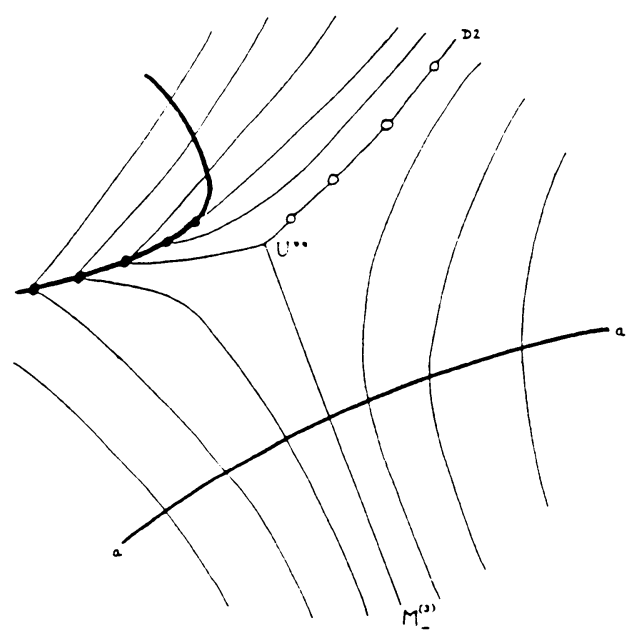

$8 A^{\prime}$

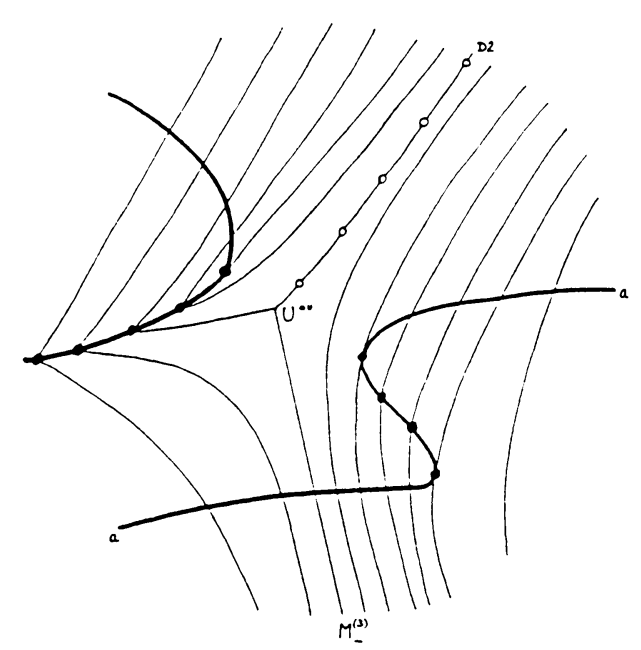

$8 B$

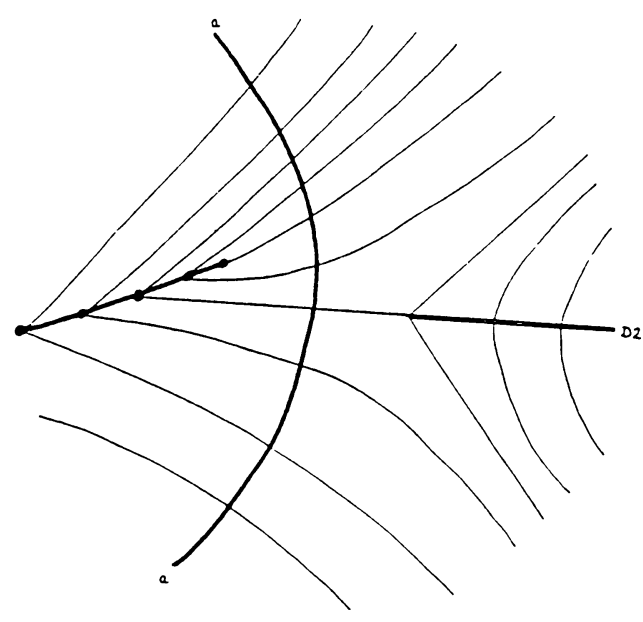

$8 C$

Figure 8. $U_{R}$ wave curves, Case II

four large sectors, labeled A, $\mathrm{A}^{\prime}, \mathrm{B}, \mathrm{C}$ in the figure, such that for $U_{L}$ within each sector, the topological structure of the wave curves remains the same. The wave curves associated to each sector in the $U_{R}$-plane are shown in Figure 8a, a', b, c; in these figures we have suppressed the location of $U_{L}$, except to indicate (by the letter "a") which part of the 1-wave curve is attached, i.e. contains $U_{L}$. (Remarks: (i) The prime indicates that, up to homeomorphism, the $U_{R}$-curves for $U_{L}$ in sectors $A$ and $A^{\prime}$ are reflections of one other. Indeed if $b=0$ in (1.3), these curves are exact reflections of one another. (ii) The more refined structure of the solution that we consider in subsection (b) may change within a large sector; e.g., between sectors A1 and A2.)

Besides the 1-wave curve and the 2-wave curves, three other kinds of curve are indicated in Figure 8. These curves, which represent singularities of the coordinate 


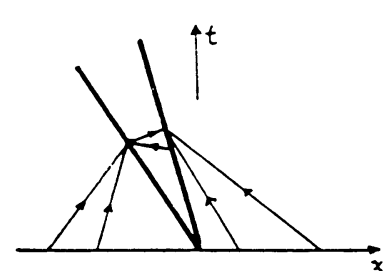

(a)

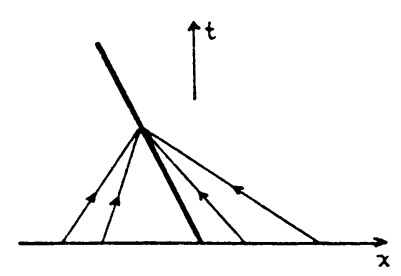

(d)

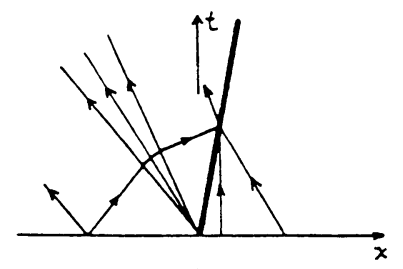

(b)

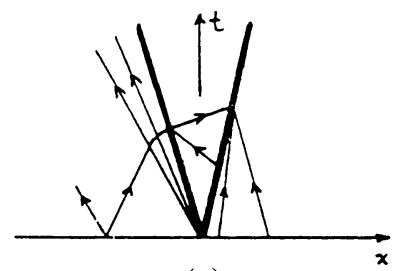

(c)

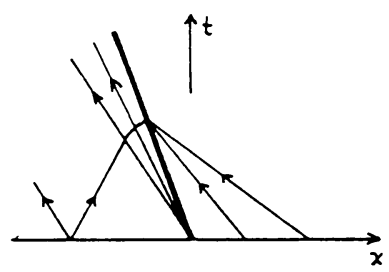

(e)

FIGURE 9. Almost overcompressive and overcompressive waves

system, arise from overcompressive waves, from secondary bifurcation, and from detached rarefaction waves. The associated curves are shown in Figure 8 as dark curves accented by solid circles, light curves accented by open circles, and parallel light lines, respectively. We describe each of these in turn.

Overcompressive waves arise as follows. Consider $U_{R}$ approaching the overcompressive wave curve in Figure 8 along the 2-wave curve $W_{2}\left(U^{*}\right)$ of some intermediate state $U^{*}$; we shall show in $\S 3$ (c) that this approach must be along a 2-shock curve. The solution of the Riemann problem for such $U_{R}$ consists of a 1-wave followed by a 2-shock; as illustrated in Figures 9a, b, and c, the 1-wave may be a shock, a rarefaction, or a composite. As $U_{R}$ approaches the overcompressive wave curve, the 2-shock merges with the 1-wave, resulting in the limit in the configurations illustrated in Figures $9 \mathrm{~d}$ and $9 \mathrm{e}$. (Figure $9 \mathrm{~d}$ is the limiting case of Figure 9a, while Figure 9e shows the limiting case of both Figures $9 b$ and 9c.) Since in Figures 9d and $9 \mathrm{e}$, more characteristics enter the shock than specified in the Lax admissibility criterion, we call such waves overcompressive. Note that the 2-wave curves in the $U_{R}$-plane stop at the overcompressive wave curve. The intermediate state $U^{*}$ used to solve the Riemann problem suffers a jump when $U_{R}$ crosses the overcompressive wave curve. Thus considered pointwise, the solution of the Riemann problem depends discontinuously on $U_{R}$ across an overcompressive wave curve. However, the solution is continuous in the $L^{1}$ norm since, as illustrated in Figure 9, the intermediate state $U^{*}$ is assumed on a wedge in the $(x, t)$-plane of vanishing thickness. (Remark: The overcompressive shocks discussed in $\S 2(\mathrm{~b})$ are one kind of overcompressive wave, namely that illustrated in Figure 9d.)

The curve associated with secondary bifurcation in Figures 8, 10 arises as follows. The 2-wave curve originating from the point on the median $M_{-}^{(j)}(j=2$ or 3 ) in Figure 8 bifurcates at point $U^{* *}$; one fork of the wave curve continues as a shock curve, while the other fork continues as the shock-rarefaction curve $D 2$. This 


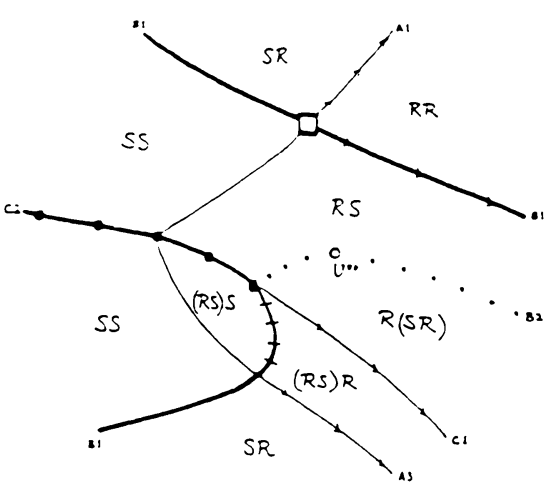

(A1)

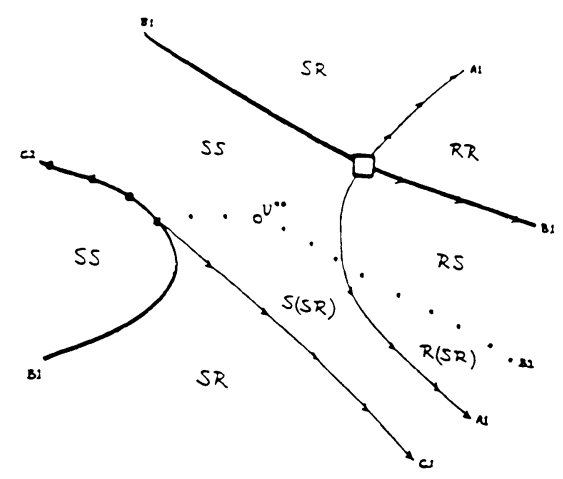

(A3)

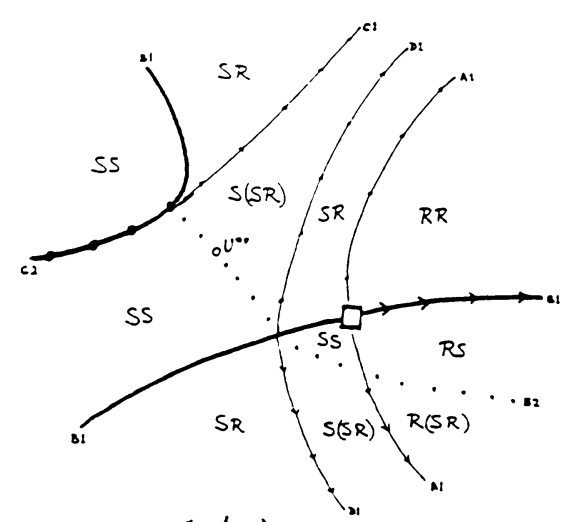

$\left(A^{\prime} 1\right)$

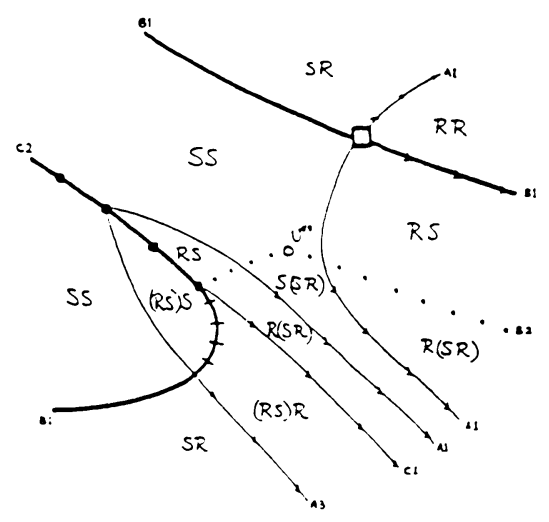

(A2)

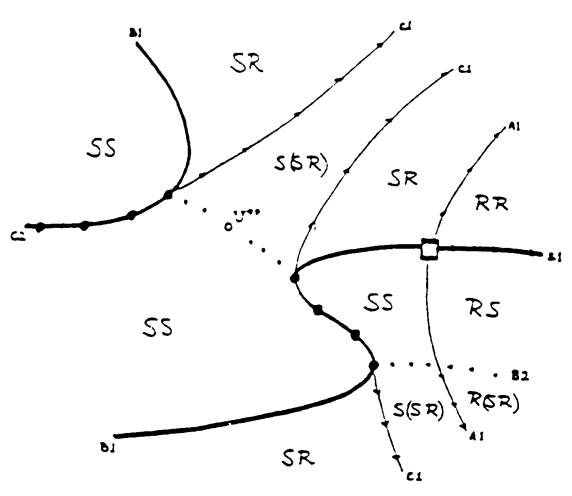

(B)

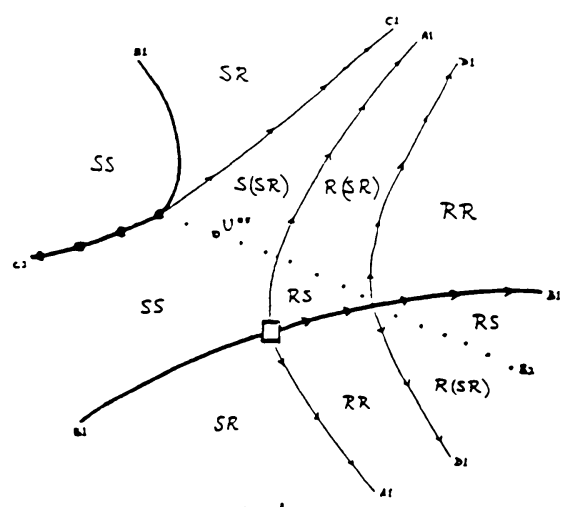

$\left(A^{\prime} 2\right)$

Figure 10. $U_{R}$-regions, Case II 


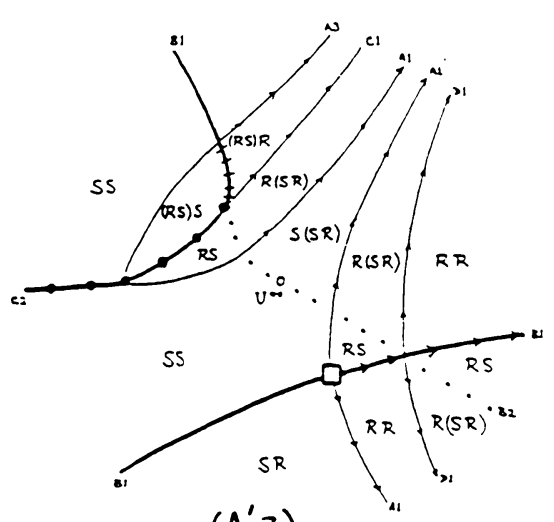

$\left(A^{\prime} 3\right)$

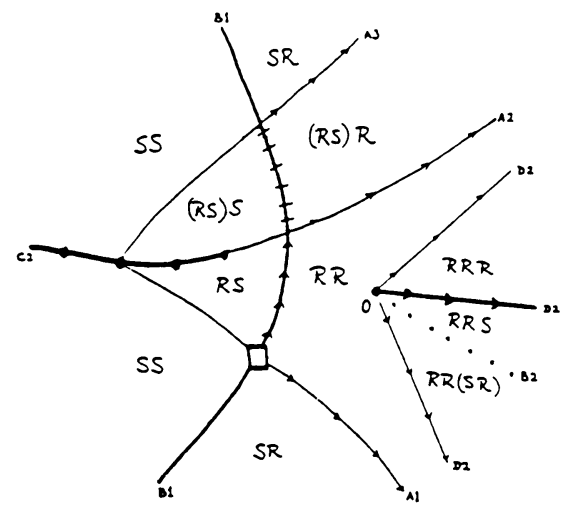

(c1)

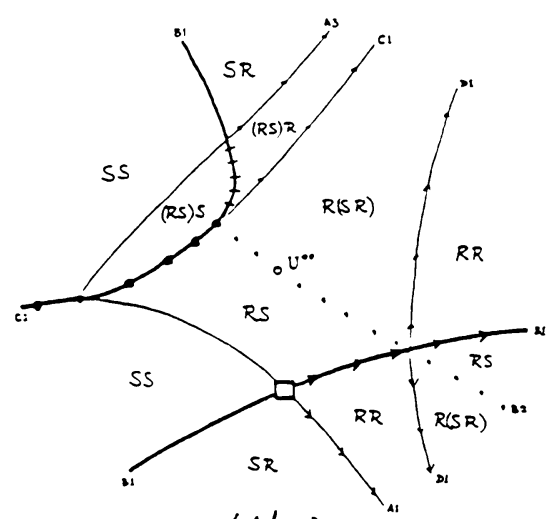

$\left(A^{\prime} 4\right)$

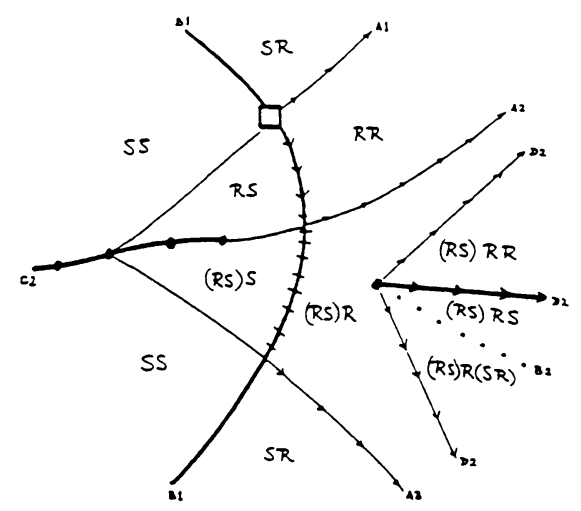

(C2)

FIgURE 10. $U_{R}$-regions, Case II (continued)

splitting of the wave curve is related to the secondary bifurcation discussed in $\$ 2$. The dependence of the solution of the Riemann problem on $U_{R}$ is continuous across $D 2$ but not differentiable. The problem is that for $U_{R}$ on both sides of $D 2$, the intermediate state $U^{*}$ on the 1-wave curve always lies to the right of $M_{-}^{(j)}$; in other words, $U^{*}$ has a singularity across $D 2$ like the absolute value function on the line.

The detached rarefaction curve is associated with a striking phenomenon; viz., for certain ranges of data $U_{L}, U_{R}$, the solution to the Riemann problem for the $2 \times 2$ system (1.3) consists of three waves separated by constant states. To explain this, consider the wave curve lying on the median $M^{(1)}$ in Figure 8C. The portion of $M_{-}^{(1)}$ between the $W_{1}$ curve and the origin is a 2-rarefaction curve, while the portion $M_{+}^{(1)}$ lying to the right of the origin is a 1-rarefaction curve. This change in families occurs at the umbilic point, where the wave speeds coincide. The detached rarefaction curve plays a role in the solution of the Riemann problem, represented in Figure 8C, when $U_{R}$ lies between $M_{-}^{(2)}$ and $M_{-}^{(3)}$. In this case, the solution consists of a 1-wave from 
$U_{L}$ to $M_{-}^{(1)}$, followed by the rarefaction of mixed character along $M^{(1)}$ as discussed above, in turn followed by a 2-wave from $M_{+}^{(1)}$ to $U_{R}$. The rarefaction curve $M_{+}^{(1)}$ is referred to as detached since it acts like a detached 1-wave curve in Figure 8C.

(b) Regions in the $U_{R}$-plane. In drawing the wave curves of Figure 8, we suppressed the distinctions between shocks, rarefactions, and composite waves. In this section we include this additional information, using the following construction: for each $U_{L}$, we divide the $U_{R}$-plane into regions such that for all $U_{R}$ in a given region, the solution to the Riemann problem involves a specific combination of shock, rarefaction, and composite waves. Figure 8 shows these $U_{R}$-regions for several representative values of $U_{L}$. The regions are labeled with a combination of $S$ 's and $R$ 's to indicate the shocks and rarefactions which occur in solving the Riemann problem; parentheses indicate a composite wave. The waves appear in the order indicated, with increasing $x / t$; of course their strengths vary (smoothly) as $U_{R}$ varies within a region.

The precise partition of the $U_{R}$-plane into regions is different for different values of $U_{L}$. However, as with the bifurcation diagrams and wave curve diagrams, it is helpful to distinguish between essential and inessential differences. If $U_{L}$ crosses one of the four major boundary rays in Figure 7 (i.e., $M_{+}^{(j)}, j=1,2,3$, or $H_{1}$ ), then the topology of the regions changes; if $U_{L}$ crosses one of the other six boundary rays in Figure 7, then the regions change in a more subtle, but still discontinuous, way; for $U_{L}$ within one of the ten sectors of Figure 7, there are no qualitative changes in the $U_{R}$-regions. In Figure 10, we show the $U_{R}$-regions for ten values of $U_{L}$, one from each of the ten sectors in Figure 7.

A lot of information is summarized in Figure 10. The key to synthesizing this information lies in understanding the phenomena which give rise to the boundaries between regions in the $U_{R}$-plane. In the subsection immediately following, we shall classify these phenomena systematically. Thus in one sense that subsection may be regarded as an extended explanation of Figure 10. Of course the boundary curves are also of interest because the solution of the Riemann problem is a piecewise smooth function of $U_{R}$ with singularities along these curves. The nature of these singularities is discussed briefly at the end of $\S 3(\mathrm{c})$.

Besides the boundaries in the $U_{R}$-plane to be studied momentarily, there are also boundaries in the $U_{L}$-plane. Figure 11 shows the $U_{R}$-regions when $U_{L}$ lies on one of the four major boundary rays, thereby clarifying the transition when $U_{L}$ crosses a major boundary. (We omit the $U_{R}$ diagrams showing the simpler transitions as $U_{I}$. crosses a minor boundary.)

(c) Boundary curves. Our discussion of boundary curves applies only to quadratic nonlinearities since we make essential use of our result that, in the quadratic case, the only composite 1-waves are rarefaction-shocks and the only composite 2-waves are shock-rarefactions. The discussion applies to Cases II-IV, although we illustrate the phenomena with examples from Case II.

Broadly speaking, boundaries of regions in the $U_{R}$-plane arise in one of four ways. The first two ways, which differ only in which characteristic family is principally involved, include phenomena that are familiar from strictly hyperbolic equations; 

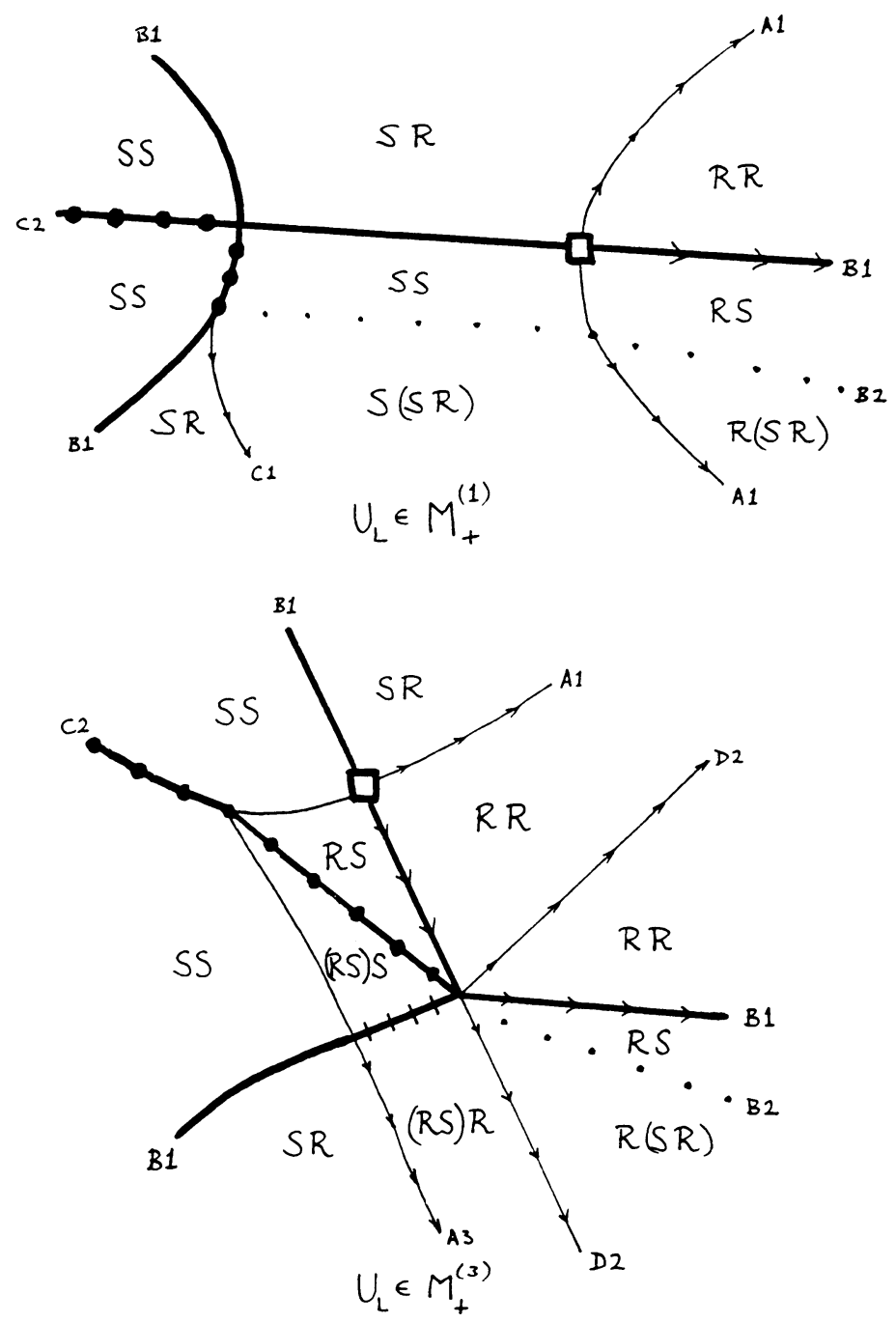

FIGURE 11

the last two, which involve both characteristic families in an essential way, include phenomena associated with the failure of strict hyperbolicity. Let us survey these four classes of boundaries before beginning our systematic study.

The first class of boundary curves arises from transition points $\hat{U}$ on $W_{1}\left(U_{L}\right)$ where there is a change in the precise combination of 1-waves represented by the wave curve; e.g., where a shock loses admissiblity at $\hat{U}$ and the wave curve continues with the rarefaction-shock construction. From each such $\hat{U}$, the 2-wave curve $W_{2}(\hat{U})$ is a boundary curve in the $U_{R}$-plane. The curves labeled A1, A2, or A3 in Figure 10 arise through this mechanism. 
TABLE $1 . U_{R}$-boundaries

\begin{tabular}{|c|c|c|c|c|}
\hline & $\begin{array}{c}\text { A } \\
\text { Transition points } \\
\text { on } W_{1}\left(U_{L}\right)\end{array}$ & $\begin{array}{c}\text { B } \\
\text { Transition points } \\
\text { on } W_{2}\left(U^{*}\right)\end{array}$ & $\begin{array}{l}\text { C } \\
\text { Phenomena involving } \\
\text { overcompressive waves }\end{array}$ & $\begin{array}{c}\mathrm{D} \\
\text { Nongeneric points on } W_{1}^{\prime}\left(U_{l}\right)\end{array}$ \\
\hline $\begin{array}{c}1 \\
\text { Boundary } \\
\end{array}$ & $\begin{array}{c}R_{1} \text { meets } S_{1} \text { at } U_{L} \\
\text { (zero strength) } \\
W_{2}\left(U_{L}\right)\end{array}$ & $\begin{array}{c}R_{2} \text { meets } S_{2} \text { at } U^{*} \\
\text { (zero strength) } \\
W_{1}\left(U_{L}\right)\end{array}$ & $\begin{array}{c}\text { (i) } S_{1} \text { meets } S_{0} \text { or } \\
\text { (ii) }(R S)_{1} \text { meets }(R S)_{0} \\
\text { at } U^{*} \\
\left.\text { [Eqn: } s=\lambda_{2}\left(U^{*}\right)\right] \\
R_{2}\left(U^{*}\right)\end{array}$ & $W_{1}$ crosses $F_{2}$ at $\hat{U}$ \\
\hline Phenomenon & $\begin{array}{l}R_{1} \text { meets }(R S)_{1} \text { at } \hat{U} \\
\left(\hat{U} \in F_{1}\right)\end{array}$ & $\begin{array}{c}S_{2}\left(U^{*}\right) \text { meets } \\
(S R)_{2}\left(U^{*}\right) \text { at } \hat{U} \\
(\text { limit point) } \\
{\left[\text { Eqn: } s=\lambda_{2}(\hat{U})\right]} \\
\\
\\
\text { (no special notation) }\end{array}$ & $\begin{array}{l}S_{2}\left(U^{*}\right) \text { ends at } \hat{U} \text { on } \\
\text { (i) } S_{0} \text { or (ii) }(R S)_{0} \\
\text { Eqn: (i) } U^{*} \in S_{1}\left(U_{L}\right) . \\
s\left(U_{l}, U^{*}\right)=s\left(U^{*}, \hat{U}\right) . \\
\text { (ii) } U^{*} \in R_{1}\left(U_{L}\right) . \\
s\left(U^{*}, \hat{U}\right)=\lambda_{1}\left(U^{*}\right) . \\
\text { (i) } S_{0}\left(U_{l}\right), \\
\text { (ii) }(R S)_{0}\left(U_{l}\right)\end{array}$ & $\begin{array}{c}W_{1} \text { crosses } M^{(1)} \\
\text { Two cases: } \\
\text { (i) } M^{(1)} \text {-secondary bifurcation } \\
\text { (ii) } M^{(1)} \text {-detached rarefaction } \\
\\
\text { (i) } R_{2}\left(U^{* *}\right)^{\dagger} \\
\text { (ii) } M^{(1)} \cup M^{(2)} \cup M^{(3)}\end{array}$ \\
\hline $\begin{array}{c}\text { Phenomenon } \\
3 \\
\text { Boundary }\end{array}$ & $\begin{array}{c}S_{1} \text { meets }(R S)_{1} \text { at } \hat{U} \\
\quad \text { overlap) } \\
{\left[\text { Eqn: } s=\lambda_{1}\left(U_{L}\right)\right]} \\
W_{2}(\hat{U})\end{array}$ & & & $\begin{array}{c}R_{1} \text { enters origin } \\
\text { (only occurs in Case III) } \\
M^{\prime \prime \prime}, j=2 \text { or } 3\end{array}$ \\
\hline
\end{tabular}

${ }^{\dagger}$ 2-wave splits at $U^{* *}$

The second class of boundary curves is similar to the first, but involves the 2-wave curves. For each $U^{*} \in W_{1}\left(U_{L}\right)$, there are transition points $\hat{U}$ on $W_{2}\left(U^{*}\right)$. The locus of these points $\hat{U}$ as $U^{*}$ moves along $W_{1}\left(U_{L}\right)$ is a $U_{R^{2}}$-boundary. The curves labeled $\mathrm{B} 1$ or $\mathrm{B} 2$ in Figure 10 arise through this mechanism.

The third class of boundary curves includes the overcompressive wave curves described in $\S 3(\mathrm{a})$ and the boundaries they generate. These boundaries are labeled $\mathrm{C} 1$ or $\mathrm{C} 2$ in Figure 10.

The fourth class of boundary curves appears when $W_{1}\left(U_{L}\right)$ crosses a $U_{L}$-boundary. Specifically, the boundaries labeled D1 in Figure 10 result from $W_{1}\left(U_{L}\right)$ crossing the 2 -inflection locus, and the boundaries labeled D2 in Figures 8 and 10 correspond to $W_{1}\left(U_{l}\right)$ crossing a median $M_{-}^{(j)}(j=1,2$ or 3$)$.

We expand on this classification of $U_{R}$-boundary curves in Table 1 , which enumerates the ways that such curves arise (for a quadratic nonlinearity). The four columns, A, B, C, D, of Table 1 correspond to the four classes above, but in Table 1 we have further subdivided each class. The labels A1, A2, A3, etc. of boundary curves in Figure 10 derive from this subdivision. In making this table we consider only generic $U_{I}$; i.e., not lying on one of the boundary rays, $M^{(j)}, L_{k}, F_{k}$, or $H_{k}$, in the $U_{L}$-plane.

The entries of column A arise from transition points on the 1-wave curve, $W_{1}\left(U_{L}\right)$. This wave curve is divided into segments representing rarefactions, shocks, and composite waves; by $\S 5$, the latter contains only rarefaction-shocks. At a transition point, two such segments meet one another. Thus there are three kinds of transition points; viz., points where rarefaction meets shock, where rarefaction meets composite, and where shock meets composite. These three possibilities correspond to entries A1, A2, and A3 in Table 1, respectively. More precisely, rarefaction meets 
shock at $U_{L}$ where both waves have zero strength; rarefaction meets composite where the rarefaction curve crosses the 1-inflection locus $F_{1}$; shock meets composite where the shock curve loses admissibility, an overlap point in the terminology of $\$ 2$. (Remark: Note that the $\mathrm{A} 1$ boundary is precisely the 2-wave curve, $W_{2}\left(U_{L}\right)$. This fact may help in interpreting Figure 10.)

The above discussion is slightly misleading as regards shock-composite transition points in that it masks some important structure. To clarify this, recall that along an admissible 1-shock curve the three inequalities (2.4), relating characteristic speeds and the shock speed, must hold. At a shock-composite transition point $\hat{U}$, one of these inequalities fails, so that one of the following equalities is satisfied.

(a) $s=\lambda_{1}\left(U_{L}\right)$

(b) $s=\lambda_{1}(\hat{U})$,

(c) $s=\lambda_{2}(\hat{U})$.

Case (a) corresponds to entry A3 in Table 1, as discussed above. It follows from our results in $\$ 5$ that case (b) does not occur for quadratic nonlinearities-otherwise there would be shock-rarefactions in the 1-characteristic family. Case (c) does occur, but since the 1-shock loses admissibility by becoming overcompressive, we include this case under entry $\mathrm{C} 1$, specifically $(\mathrm{C} 1)_{\mathrm{i}}$, the first of two subcases. Note that in this case the apparent continuation of $W_{1}\left(U_{L}\right)$ beyond the transition point $\hat{U}$ is the overcompressive shock curve $S_{0}\left(U_{L}\right)$. Since $S_{0}\left(U_{L}\right)$ is not part of $W_{1}\left(U_{L}\right)$, we see that, strictly speaking, the 1 -wave curve stops at $\hat{U}$.

Incidentally, $(\mathrm{C} 1)_{\mathrm{ii}}$ describes a related situation in which the 1-wave curve again stops at a point $\hat{U}$, but in the latter situation a 1-rarefaction-shock, rather than a 1 -shock, loses admissibility by becoming overcompressive. In both cases $(\mathrm{C} 1)_{\mathrm{i}}$ and $(\mathrm{C} 1)_{\mathrm{ii}}$, the 2-rarefaction curve $R_{2}(\hat{U})$ is a $U_{R}$-boundary. Although the notations in Figure 10 do not distinguish between cases $(\mathrm{C} 1)_{\mathrm{i}}$ and $(\mathrm{C} 1)_{\mathrm{ii}}$, the following property distinguishes them: the two regions on either side of a $(\mathrm{C} 1)_{\mathrm{i}}$ boundary are labeled $S R$ and $S(S R)$, while the regions for a $(\mathrm{C} 1)_{\mathrm{ii}}$ boundary are labeled $(R S) S$ and $R(S R)$. The shocks involved in these regions undergo an interesting transition as $U_{R}$ crosses the $\mathrm{C} 1$ boundary $R_{2}(\hat{U})$. Across the $(\mathrm{C} 1)_{\mathrm{i}}$ boundary, the 1 -shock splits into a 1 -shock and a 2-shock, while across the $(\mathrm{C} 1)_{\mathrm{ii}}$ boundary, the shock changes from a 1 -shock to a 2 -shock. A second noteworthy feature is that the intermediate state $U^{*}$ needed to solve the Riemann problem jumps disontinuously when $U_{R}$ crosses the boundary $R_{2}(\hat{U})$. For example in Figure 10.A1, if $U_{R}$ belongs to the region labeled $R(S R)$, then $U^{*}$ lies on the branch of $W_{1}\left(U_{L}\right)$ connected to $U_{L}$, but if $U_{R}$ belongs to $(R S) R$, then $U^{*}$ lies on the detached branch of $W_{1}\left(U_{L}\right)$.

The consideration of column B is similar to that of a column A. Transition points on 2-wave curves $W_{2}$ occur where rarefaction meets shock, where rarefaction meets composite, or where shock meets composite. The first possibility corresponds to entry B1. The second possibility does not occur since there are no rarefaction-shock composite 2-waves. The third possibility may be divided into three cases according to which inequality in (2.5) becomes an equality; one case correspoinds to entry B2, one case is ruled out by $\S 5$, and one case corresponds to entry $(\mathrm{C} 2)_{\mathrm{ii}}$. (Remark: B2 boundaries are shown as dotted in Figure 10; these are the only boundaries which are not themselves 1-wave, 2-wave, or overcompressive wave curves.) 
Column $\mathrm{C}$ lists boundary curves resulting from overcompressive waves. Of the two entries in this column, we have already encountered $\mathrm{C} 1$ in discussing column $\mathrm{A}$. We saw that this kind of boundary was generated by a point where the 1-wave curve stops by virtue of becoming overcompressive. Here we shall focus on entry $\mathrm{C} 2$, at which 2-wave curves stop.

The boundaries listed in columns A and B occur when wave curves of one family, considered individually, lose admissibility and need to be continued by a different construction. In the case of a boundary described by entry $\mathrm{C} 2$, either wave separately is admissible, but together they are not. Specifically, consider the situation of $\S 3(\mathrm{a})$ in which overcompressive waves occurred; i.e., consider fixed $U_{L}$ and $U^{*} \in W_{1}\left(U_{L}\right)$ such that as the wave amplitude along $W_{2}\left(U^{*}\right)$ increases, the 2-wave speed slows down to the extent that the 2-wave and 1-wave collide with one another in the $(x, t)$-plane (cf. Figure 9). At such a point we may say that the two waves, considered together, lose admissibility; moreover, no continuation is possible.

REMARK: We now prove our claim of $\S 3\left(\right.$ a) that in this situation $W_{2}\left(U^{*}\right)$ must be a shock curve near the boundary in question. In general, the 2-wave includes shocks, rarefactions, and shock-rarefaction composites. However, along a rarefaction curve or a shock-rarefaction curve, the characteristic speed increases with increasing amplitude, so an overcompressive wave cannot be encountered.

Entry $\mathrm{C} 2$ includes two subcases, $(\mathrm{C} 2)_{\mathrm{i}}$ and $(\mathrm{C} 2)_{\mathrm{ii}}$, corresponding to the two overcompressive waves illustrated in Figures $9 \mathrm{~d}$ and e, respectively. One difference between these two cases is that in case $(\mathrm{C} 2)_{\mathrm{i}}$, the boundary curve (i.e., the overcompressive wave curve) is part of the Hugoniot locus of $U_{L}$; viz., $S_{0}\left(U_{L}\right)$. By contrast, in case $(\mathrm{C} 2)_{\mathrm{ii}}$, the overcompressive wave curve involves a composite construction; as suggested by Figure 9e, we shall denote this portion of the overcompressive wave curve by $(R S)_{0}\left(U_{L}\right)$. In terms of Figure 10, another difference between the two cases may be seen by varying $U_{R}$ near the boundary curve. In case $(\mathrm{C} 2)_{i}$, for values of $U_{R}$ on either side of $S_{0}\left(U_{L}\right)$, the solution of the Riemann problem has the structure illustrated in Figure $9 \mathrm{a}$, although the intermediate state $U^{*}$ jumps as $U_{R}$ crosses the overcompressive wave curve. By contrast, in case (C2) $)_{\mathrm{ii}}$, the solution of the Riemann problem resembles Figure $9 \mathrm{~b}$ for $U_{R}$ on one side of $(R S)_{0}\left(U_{L}\right)$ and resembles Figure $9 \mathrm{c}$ for $U_{R}$ on the other side. Thus in Figure 10, if the two regions on either side of a segment of a $\mathrm{C} 2$ boundary are both labeled $S S$, then that segment corresponds to entry $(\mathrm{C} 2)_{\mathrm{i}}$; if one region is labeled $R S$ and the other $(R S) S$, then that segment corresponds to $(\mathrm{C} 2)_{\mathrm{ii}}$.

Columns A, B, and C list boundary curves in the $U_{R}$-plane related to changes in the admissibility of waves. Boundaries in the $U_{R}$-plane also arise through one further mechanism. Although we consider generic $U_{I}$, nongeneric points occur on $W_{1}\left(U_{I}\right)$ when this wave curve crosses a boundary in the $U_{L}$-plane. The $U_{R}$-boundaries that such points generate are listed in column D. Although this column contains three entries, only the first two are relevant to Case II. (The third entry, D3, is self explanatory. See Figures $18 \mathrm{C}, \mathrm{C}^{\prime}$, D of Appendix 2.) One might have expected four entries in column D for Case II, rather than two, corresponding to the four classes of $U_{I}$-boundaries: viz., $M, L, F$, and $H$. However, it turns out that when $W_{1}\left(U_{L}\right)$ crosses either $L$ or $H$, only boundaries already listed in column $\mathrm{C}$ are generated. 
Entry D1 applies when $W_{1}\left(U_{L}\right)$ crosses the 2-inflection locus $F_{2}$. Even in the strictly hyperbolic case, $W_{1}\left(U_{L}\right)$ may cross $F_{2}$; thus the boundaries described by entry D1 are not new, although the fact that Liu [11] emphasizes wave curves rather than regions may make them appear so. Note (e.g., in Figure 10A') that three distinct boundary curves pass through a point $\hat{U}$ where $W_{1}\left(U_{L}\right)$ crosses $F_{2}$, only one of which is not listed elsewhere in Table 1 . The curve $\mathrm{B} 1$ is $W_{1}\left(U_{L}\right)$ itself; the curve B2 also passes through $\hat{U}$; the curve D1 is the new boundary generated by the crossing. In symbols, the new boundary is $W_{2}(\hat{U})$.

REMARK. If a segment of $W_{1}\left(U_{L}\right)$ corresponding to rarefaction waves crosses the other inflection locus $F_{1}$, a $U_{R}$-boundary curve may also be generated. Specifically, if a segment of $W_{1}\left(U_{L}\right)$ representing rarefaction waves crosses $F_{1}$ and if the characteristic speed along $W_{1}\left(U_{L}\right)$ has a local maximum, then the rarefaction curve must be continued with the rarefaction-shock construction. The boundaries resulting from such transitions are already listed in entry A2.

Entry D2 applies when $W_{1}\left(U_{L}\right)$ crosses a median $M_{-}^{(j)}, j=1,2,3$. (Crossings of $M_{+}^{(j)}$ do not generate $U_{R^{-}}$-boundaries.) This entry is divided into two subcases according as $j=2,3$ or $j=1$. The former corresponds to secondary bifurcation, the latter to the detached rarefaction curve, both of which phenomena were discussed in \$3(a). In the case of secondary bifurcation, the boundary is the rarefaction curve $R_{2}\left(U^{* *}\right)$, where $U^{* *}$ is the point at which secondary bifurcation occurs. These boundaries are not drawn in Figure 10 since the solution of the Riemann problem has the same decomposition into waves for $U_{R}$ on either side of the curve; however, $U^{* *}$ is shown in the figure, and by comparing with nearby rarefaction curves, one can locate the boundary in question. In the case of the detached rarefaction curve, the $U_{R}$-boundaries consist of the two half rays $M_{-}^{(2)}$ and $M_{-}^{(3)}$ as well as the detached rarefaction curve $M_{+}^{(1)}$ (cf. Figure 10c).

We conclude our discussion of Table 1 by relating the classification of $U_{R^{-}}$ boundaries to another issue mentioned earlier; viz., that the solution of the Riemann problem is a piecewise smooth function of $U_{R}$ with singularities on the $U_{R}$-boundaries. Across the boundaries in column $\mathrm{C}$, the solution is discontinuous (considered pointwise-it is continuous in the $L_{1}$ sense). Across the boundaries in entry D2, the solution is continuous but its first derivative is not. (Remarks: Recall that the boundaries described by entry (D2) $)_{i}$ are not shown in Figure 8 . Regarding entry (D2) ${ }_{\mathrm{ii}}$, the first derivative of the solution is discontinuous across $M_{-}^{(2)}$ and $M_{-}^{(3)}$, but across $M_{+}^{(1)}$ its behavior is smoother.) Across the boundaries in entry D1 or in columns $\mathrm{A}$ or $\mathrm{B}$, the first derivative of the solution is continuous but some higher derivative is not (cf. [11]).

4. Characterization of $U_{L}$-boundaries. The two basic ingredients in piecing together the $U_{R}$-regions that solve the Riemann problem for fixed $U_{L}$ are the rarefaction curves given in detail in Figure 2 and discussed in [13], and the Hugoniot loci. The rarefaction curves through a given $U_{L}$ change with $U_{L}$ in a way that is easily understood from Figure 2 . The only $U_{L}$-boundaries that have a profound significance on the rarefaction curves are the inflection loci $F_{k}(k=1$ or 2$)$, at which the 
orientation of the rarefaction curve $R_{k}$ changes. In this section we concentrate on significant changes in the Hugoniot loci as $U_{L}$ varies, by deriving properties of the bifurcation diagrams for equation (2.2). These changes take place as $U_{L}$ crosses one of the four types of $U_{L}$ boundaries labeled $F, M, L, H$ in Figure 3. For $F, M$ and $L$ we give explicit equations to be satisfied by $U_{L}$, and describe in a series of Lemmas the special features of the Hugoniot locus associated with each of these boundaries. For the hysteresis locus $H$, however, the description lacks an explicit equation.

The inflection loci $F_{k}\left(k=1\right.$ or 2) consist of values of $U_{L}$ for which equation (1.3) loses genuine nonlinearity in the $k$ th characteristic field. We express this in two ways:

$$
d \lambda_{k} \cdot r_{k}=0=d^{2} Q\left(r_{k}, r_{k}\right) \cdot r_{k} \text { at } U_{L} .
$$

Note that by Euler's relation for homogeneous functions, the second equation of (4.1) is $C\left(r_{k}\left(U_{L}\right)\right)=0$. This leads to the following explicit formulae for the inflection loci: $b u+v=0$, in Cases I-IV and $\left(\left(c^{2}-1\right)+b c\right) v=\left((1-a) c+\left(1-c^{2}\right) b\right) u$, in Case I, where $c=\left(-b \pm \sqrt{\left(b^{2}-4 a / 3\right)}\right) / 2$. As discussed in $\$ 2$, the RankineHugoniot condition

$$
-s\left(U-U_{L}\right)+Q(U)-Q\left(U_{L}\right)=0
$$

is regarded as a bifurcation problem for $U$, with bifurcation parameter $s$, auxiliary parameters $U_{L}$ and trivial solution $U=U_{L}$ (for any $s$ ). Provided $d Q(U)$ has distinct (real) eigenvalues for each $U \neq 0$ (as is true for $a \neq 1+b^{2}$ ), bifurcation from the trivial solution $U=U_{L} \neq 0$ takes place at $s=\lambda_{k}\left(U_{L}\right)(k=1$ or 2$)$. Moreover, for each of $k=1, k=2$, the nontrivial solutions $(s, U)$ near $\left(\lambda_{k}\left(U_{L}\right), U_{L}\right)$ form a curve $\{(s=s(\varepsilon), U=U(\varepsilon))\}$ parameterized smoothly by $\varepsilon$, and called a primary branch. Taking $\varepsilon=0$ to correspond to the primary bifurcation point, we have

$$
\begin{array}{ll}
s(0)=\lambda_{k}\left(U_{L}\right), & U(0)=U_{L}, \\
\dot{s}(0)=\frac{1}{2} \lambda_{k}\left(U_{L}\right), & \dot{U}(0)=r_{k}\left(U_{L}\right),
\end{array}
$$

where we use a dot to denote $d / d \varepsilon$. This result is summarized in [10], and proved in detail in [2] using the implicit function theorem.

When $\dot{s}(0) \neq 0$, the bifurcation is said to be transcritical (the bifurcation point being the "critical" point). Since

$$
\dot{\lambda}_{k}\left(U_{L}\right)=d \lambda_{k}\left(U_{\lambda}\right) \cdot r_{k}\left(U_{L}\right),
$$

we have transcritical bifurcation providing $U_{L} \notin F_{k}$.

If $\dot{s}(0)=0$ (i.e., $U_{L} \in F_{k}$ ), the bifurcation will be one-sided if $\ddot{s}(0) \neq 0$, namely supercritical if $\ddot{s}(0)>0$ (since then $s(\varepsilon)>\lambda_{k}\left(U_{L}\right)$ for small $\varepsilon \neq 0$ ) and subcritical if $\ddot{s}(0)<0$. As might be guessed from (4.3), there is a connection between $\ddot{s}(0)$ and $\ddot{\lambda}_{k}\left(U_{L}\right)$. But we can read off the sign of $\ddot{\lambda}_{k}\left(U_{L}\right)$ when $U_{L} \in F_{k}$ from our knowledge of the rarefaction curves (Figure 2), and the fact that (since $\left.\dot{U}(0)=r_{k}\left(U_{L}\right)\right), d / d \varepsilon$ at $\varepsilon=0$ is the same as taking a directional derivative along $R_{k}$ at $U_{L}$. Specifically, we have for $U_{L} \in F_{k}$,

$$
\ddot{\lambda}_{k}\left(U_{L}\right) \text { is }\left[\begin{array}{ll}
<0 & \text { if } k=1, \\
>0 & \text { if } k=2,
\end{array}\right.
$$

(i.e., $\lambda_{1}$ has a maximum on $R_{1}$ at $F_{1}, \lambda_{2}$ has a minimum on $R_{2}$ at $F_{2}$ ). 
LEMMA 4.1. For $U_{L} \in F_{k}(k=1$ or 2$)$, the primary bifurcation from $(s, U)=$ $\left(\lambda_{k}\left(U_{L}\right), U_{L}\right)$ is subcritical if $k=1$, and supercritical if $k=2$.

Proof. Along the primary branch consider all quantities $s, U, \lambda_{k}, r_{k}$ as functions of $\varepsilon$. To relate $\ddot{s}$ to $\ddot{\lambda}_{k}$, we differentiate the identities (4.2) and

$$
d Q(U) r_{k}=\lambda_{k} r_{k}
$$

repeatedly with respect to $\varepsilon$ at $\varepsilon=0$, and use (4.3). The first derivative is

$$
d^{2} Q\left(r_{k}, r_{k}\right)+d Q(u) \dot{r}_{k}=\dot{\lambda}_{k} r_{k}+\lambda_{k} \dot{r}_{k} \text {. }
$$

Thus at $\varepsilon=0, \dot{\lambda}_{k}=0$ and

$$
\dot{r}_{k}=\beta r_{j} \quad \text { with } \beta=r_{j} \cdot d^{2} Q\left(r_{k}, r_{k}\right) /\left(\lambda_{k}-\lambda_{j}\right) .
$$

Here $j=3-k$, and we have normalized $r_{k}$ :

$$
r_{k} \cdot r_{k}=1 \quad(k=1,2) .
$$

Differentiating (4.7) with respect to $\varepsilon$, we find

$$
3 d^{2} Q\left(r_{k}, \dot{r}_{k}\right)+d Q(U) \ddot{r}_{k}=2 \dot{\lambda}_{k} \dot{r}_{k}+\lambda_{k} \ddot{r}_{k}+\ddot{\lambda}_{k} r_{k},
$$

from which we get at $\varepsilon=0$,

$$
\begin{aligned}
\ddot{\lambda}_{k} & =3 r_{k} \cdot d^{2} Q\left(r_{k}, \dot{r}_{k}\right) \\
& =3\left(r_{k} \cdot d^{2} Q\left(r_{k}, r_{j}\right)\right)\left(r_{j} \cdot d^{2} Q\left(r_{k}, r_{k}\right)\right) /\left(\lambda_{k}-\lambda_{j}\right) .
\end{aligned}
$$

Differentiating (4.2) twice with respect to $\varepsilon$ at $\varepsilon=0$ leads to

$$
\lambda_{k} \ddot{U}=d Q\left(U_{L}\right) \ddot{U}+d^{2} Q\left(r_{k}, r_{k}\right) \text { at } \varepsilon=0 .
$$

Now using (4.1), and reparameterizing so that $\ddot{U} \cdot r_{k}=0$, we have

$$
\ddot{U}(0)=\alpha r_{j}\left(U_{L}\right) \quad \text { with } \alpha=r_{j} \cdot d^{2} Q\left(r_{k}, r_{k}\right) /\left(\lambda_{k}-\lambda_{j}\right) \text {. }
$$

Finally, we calculate $\ddot{s}$ by differentiating (4.2) a third time with respect to $\varepsilon$ at $\varepsilon=0$, and using (4.3), (4.12):

$$
\begin{aligned}
\ddot{s} & =\left(r_{k} \cdot d^{2} Q\left(r_{k}, r_{j}\right)\right)\left(r_{j} \cdot d^{2} Q\left(r_{k}, r_{k}\right)\right) /\left(\lambda_{k}-\lambda_{j}\right) \\
& =\ddot{\lambda}_{k} / 3 .
\end{aligned}
$$

This, together with (4.5), completes the proof.

The second type of $U_{L}$-boundary we consider is the median denoted by $M_{ \pm}^{(j)}$ in Figure 3. The medians are wave curves through the umbilic point. Setting $U_{L}=0$ in the Rankine-Hugoniot condition (4.2) and taking the inner product with $U^{\perp}$, we conclude that medians are characterized by the equation

$$
U^{\perp} \cdot Q(U)=0 \text {. }
$$

Equation (4.14) leads to the cubic equation $m^{3}+2 b m^{2}+(a-2) m-b=0$ for the slopes $m=v / u$ of the medians. There are three medians in Cases I-III, and there is one median in Case IV. For quadratic nonlinearities, the medians play an additional role, namely that they constitute the set of $U_{L}$ for which there is a secondary bifurcation in the bifurcation diagram for (4.2). This is proved as Lemma 4.2 below, but we emphasize that this double role of the median is peculiar to quadratic 
nonlinearities. In general, the $U_{L}$-boundaries characterizing secondary bifurcation will not be wave curves. Let $U_{L} \neq 0$ satisfy (4.14). Then for each $\alpha \in \mathbf{R}$, the point $U=\alpha U_{L}$. satisfies (4.2) for some $s$, since the left-hand side is a multiple of $Q\left(U_{L}\right)$. Thus the entire median is part of the Hugoniot locus. Since the median includes $U_{L}$, it corresponds to one of the two primary branches. It is easy to check that if $U_{L} \in M_{+}^{(j)}$, then $s=\lambda_{2}\left(U_{L}\right)$ at the primary bifurcation point, while if $U_{L} \in M_{-}^{(j)}$, then $s=\lambda_{1}\left(U_{L}\right)$ at the primary bifurcation point. The term secondary bifurcation refers to a bifurcation point $(S, U)$ on this primary branch, with $U \neq U_{L}$. This is illustrated in Figure 6.

Lemma 4.2. $U_{L} \in \cup_{j} M^{(j)}$ and $U_{L} \neq 0$ if and only if there is a secondary bifurcation point $(s, U)$ in the bifurcation diagram. Moreover, $U$ and $U_{L}$ lie on opposite sides of the origin on the same median $M^{(j)}$.

Proof. Let $Q(u, v)=d C(u, v)$, where $C(u, v)=a u^{3} / 3+b u^{2} v+u v^{2}$. Eliminating $s$ from (4.2), we can relate $U=(u, v)$ to $\left(u_{L}, v_{L}\right)$ through the formula

$$
\left[\begin{array}{l}
u \\
v
\end{array}\right]=\left[\begin{array}{l}
u_{L} \\
v_{L}
\end{array}\right]+\frac{2}{(m-\eta)}\left[\begin{array}{cc}
\eta & -1 \\
m \eta & -m
\end{array}\right]\left[\begin{array}{l}
u_{L} \\
v_{L}
\end{array}\right]
$$

where

$$
m=\left(v-v_{L}\right) /\left(u-u_{L}\right),
$$

and

$$
\begin{aligned}
\eta & =\left(b+(1-a) m-b m^{2}\right) /\left(m^{2}+b m-1\right) \\
& =\left(v+v_{L}\right) /\left(u+u_{L}\right) .
\end{aligned}
$$

Thus $(u, v)$ is parameterized by $m$ providing $\eta \neq m$. Consequently, there can be no secondary bifurcation unless $\eta=m$. Now $\eta=m$ describes an asymptote for $(u, v)$ unless

$$
\eta u_{L}=v_{L} .
$$

From (4.16)-(4.18) and $\eta=m$ we get

$$
\frac{v+v_{L}}{u+u_{L}}=\frac{v-v_{L}}{u-u_{L}}=\frac{v_{L}}{u_{L}}=m
$$

and

$$
m^{3}+2 b m^{2}+(a-2) m-b=0 .
$$

But (4.20) is precisely the condition (4.14) for $\left(u_{L}, v_{L}\right)$ to lie on a median (with $m=v_{L} / u_{L}$ from (4.19)).

We have shown that if there is a secondary bifurcation point $(s, U)$, then $U_{L}$ lies on a median, and from (4.19) it follows that $U=(u, v)$ lies on the same median. That $U$ and $U_{I}$, lie on opposite sides of the origin, and that if $U_{L}$ lies on a median then there is a secondary bifurcation, are straightforward calculations, which are most easily carried out when $b=0$ (which simplifies (4.20)). We leave the details to the reader. 
Next we prove the connection between limit point-overlaps and the sets $\left\{U: \lambda_{k}(U)=0\right\}$. We shall need the following midpoint rule [7].

LEMmA 4.3. If $U_{L}, U=U_{R}$, s satisfy the Rankine-Hugoniot condition (4.2), then for $k=1$ or 2 ,

$$
s=\lambda_{k}\left(\left(U_{L}+U_{R}\right) / 2\right)
$$

and the line segment $\overrightarrow{U_{L} U_{R}}$ is tangent to the $k$-rarefaction curve at $\left(U_{L}+U_{R}\right) / 2$, unless $U_{L}=-U_{R}$.

Proof [7]. We rewrite (4.2) using the Mean Value Theorem:

$$
\begin{aligned}
s\left(U_{R}-U_{L}\right) & =Q\left(U_{R}\right)-Q\left(U_{L}\right)=\int_{0}^{1} d Q\left(U_{L}+\tau\left(U_{R}-U_{L}\right)\right) d \tau\left(U_{R}-U_{L}\right) \\
& =d Q\left(\left(U_{R}+U_{L}\right) / 2\right)\left(U_{R}-U_{L}\right)
\end{aligned}
$$

since $d Q(U)$ is linear in $U$. Hence the result.

Recall that the set $L_{k}(k=1$ or 2$)$ consists of those $U_{L}$ for which the solution set of (4.2) contains a limit point-overlap $(s, U)$. I.e. $(s, U)$ satisfies (4.2), $\dot{s}=0$ (along the bifurcation curve) and $s=\lambda_{k}\left(U_{L}\right)$.

LemMA 4.4. Let $4 a>3 b^{2}$ in equations (1.3), (1.4). Then $L_{k}=\left\{U_{L}: \lambda_{k}\left(U_{L}\right)=0\right\}$. For $U_{L} \in L_{k}$, the corresponding limit point-overlap is $(s, U)=\left(0,-U_{L}\right)$.

Proof. Let $U_{L} \in L_{k}$, and let $\left(s, U_{R}\right)$ be the corresponding limit-overlap point in the bifurcation diagram. Then $s=\lambda_{k}\left(U_{L}\right)$. From Lemma 4.3, $s=\lambda_{p}\left(\left(U_{L}+U_{R}\right) / 2\right)$ $p=1$ or 2 , and since $\dot{s}=0$, we also have $s=\lambda_{q}\left(U_{R}\right), q=1$ or 2 . Thus $U_{L}, U_{R}$ and $\left(U_{L}+U_{R}\right) / 2$ all lie in the set $\Lambda_{c}=\left\{U: \lambda_{j}(U)=c, j=1\right.$ or 2$\}$, which is a hyperbola for $c \neq 0$ and the union of two straight lines when $c=0$. (The level curves of $\lambda_{j}$ are shown in Figure 12.) However, the straight line $\overrightarrow{U_{L} U_{R}}$ can intersect a nondegenerate hyperbola in at most two points. Thus we conclude from the midpoint rule that $c=0$. To see that $U_{R}=-U_{L}$, first note that, away from $U=0$, the rarefaction curves cross the level set $\Lambda_{0}$ transversally. Thus the line segment $\overrightarrow{U_{L} U_{R}}$ cannot be tangent to any rarefaction curve, which implies $U_{R}=-U_{L}$. This completes the proof.

The equations $\lambda_{k}(u, v)=0$ reduce to the quadratic equation $m^{2}+b m+b^{2}-a$ $=0$ for the slopes $m$ of the lines $L_{1} \cup L_{2}$.

Finally, we discuss the hysteresis locus $H$ in the $U_{L}$-plane. By Lemma 4.2, if $U_{L} \notin U_{j} M_{ \pm}^{(j)}$, then there is no secondary bifurcation for equation (4.2). Thus solutions $(s, U)$ can be smoothly parameterized locally. $H_{k}$ is defined to be the set of $U_{L}$ for which there is a point $(s, U)$ in the bifurcation diagram at which

$$
\dot{s}=\ddot{s}=0,
$$

and $\dot{U}$ is parallel to $r_{k}(U)$. (Recall that $\dot{s}=0$ implies $\dot{U}$ is parallel to $r_{1}$ or $r_{2}$.)

LEMMA 4.5. $U_{L} \in H_{k}(k=1$ or 2$)$ if and only if there is a point $(s, U)$ satisfying (4.2) such that $\dot{s}=0$ and $U \in F_{k}$. That is, $H_{k}$ is the set of $U_{L}$ for which the bifurcation diagram has a limit point whose projection onto the U-plane lies on the inflection locus $F_{k}$. 
Proof. Differentiating (4.2) with respect to $\varepsilon$, we have

$$
d Q(U) \dot{U}=\dot{s}\left(U-U_{L}\right)+s \dot{U} .
$$

Thus, $\dot{s}=0$ implies $s=\lambda_{k}(U)$ and $\dot{U}=r_{k}(U)$, for $k=1$ or $k=2$. Differentiating (4.23) at this point, we have

$$
d^{2} Q\left(r_{k}, r_{k}\right)+d Q(U) \ddot{U}=\ddot{s}\left(U-U_{L}\right)+\lambda_{k} \ddot{U} .
$$

Therefore,

$$
r_{k} \cdot d^{2} Q\left(r_{k}, r_{k}\right)=\ddot{s} \cdot r_{k} \cdot\left(U-U_{L}\right) .
$$

Now $r_{k} \cdot\left(U-U_{L}\right) \neq 0$ if $U \neq U_{L}$ lies in the Hugoniot locus (this follows from the proof of Lemma 4.3, see [7]). Moreover, the left-hand side of (4.25) is zero if and only if $U \in F_{k}$ (see (4.1)). Therefore, the lemma follows from (4.25).

5. Composite waves for quadratic nonlinearities. In this section, our main task is to establish (in Theorem 5.1) which composite waves can arise for system (1.3). (This theorem covers Case I, as well as Cases II-IV.) We also discuss the Lax shock admissibility condition and Liu's generalization of Oleinik's condition $(E)$, in the light of Theorem 5.1.

THEOREM 5.1. For quadratic nonlinearities (i.e. equation (1.3)), the only composite 1-waves are rarefaction-shocks, and the only composite 2-waves are shock-rarefactions.

Proof. As in earlier sections, we use letters $R, S$ to refer to rarefaction waves and shock waves, respectively. These are called elementary waves, in contrast to composite waves, which involve combinations of elementary waves of a single family. Note that the Lax admissibility criterion dictates that in a composite wave, the elementary waves touch one another; i.e., they are not separated in the $(x, t)$-plane by constant values of $U(x, t)$.

In a series of lemmas we prove that there are no $S R$ 1-waves and no $R S$ 2-waves. This result shows that the only composite waves involving two elementary waves are precisely those specified in the theorem. But the exclusion of $S R$ 1-waves and $R S$ 2-waves also prevents all composite waves involving three or more elementary waves, thus proving the theorem. This last step follows from the exclusion of the combinations $R S R$ and $S R S$ in composite waves, since both of these include $R S$ and $S R$ composite waves.

The treatment of $S R 1$-waves and $R S$ 2-waves is similar, and we concentrate on the 1-waves. An $S R$ 1-wave involves a 1-shock whose speed is characteristic on the right. That is, if the shock joins states $U_{L}$ and $U_{R}$ with speed $s=s\left(U_{I}, U_{R}\right)$, then

$$
s\left(U_{L}, U_{R}\right)=\lambda_{1}\left(U_{R}\right) \leqslant \lambda_{1}\left(U_{L}\right) .
$$




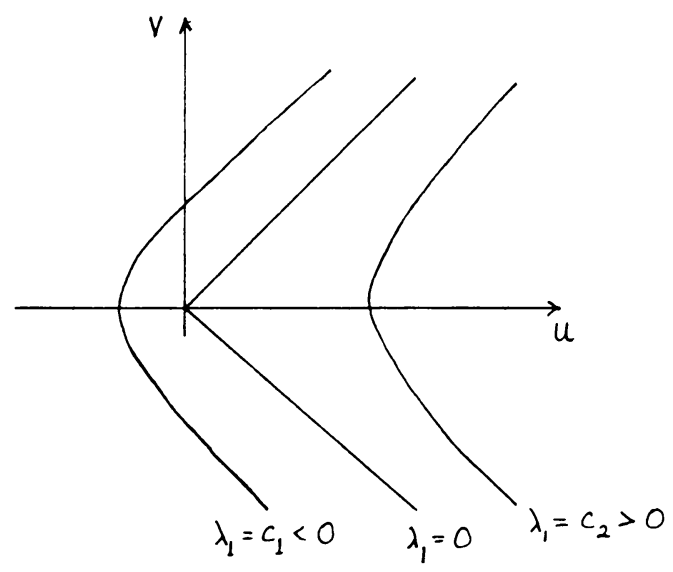

Figure 12. Level curves of $\lambda_{1}$, Cases II-IV

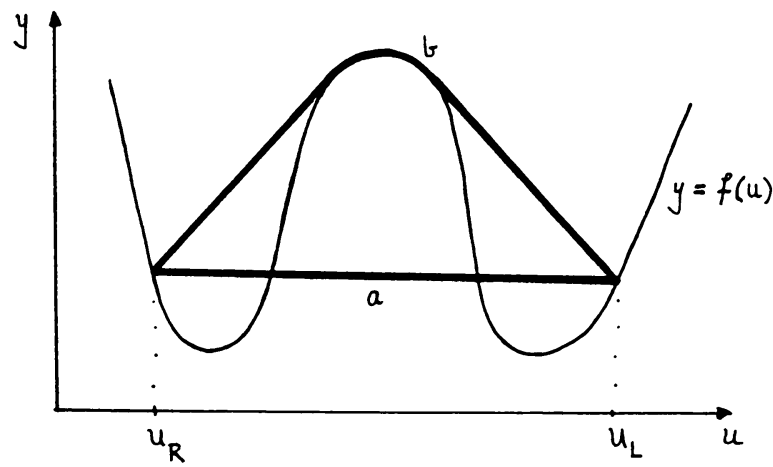

FIGURE 13. Two solutions of the Riemann problem for $u_{t}+f(u)_{x}=0$

$a$ : shock (fails condition (E))

$b$ : shock-rarefaction-shock

Lemma 5.2. For any fixed $c$, the set $K_{c}=\left\{U: \lambda_{1}(U) \geqslant c\right\}$ is convex.

Proof. The formula for $\lambda_{1}(u, v)$ is

$$
\lambda_{1}(u, v)=2\left\{(a+1) u+b v-\sqrt{((a-1) u+b v)^{2}+4(b u+v)^{2}}\right\}
$$

Thus, for $a>3 b^{2} / 4, \lambda_{1}(u, v)=c$ is one half of a hyperbola, except when $c=0$, in which case the zero set of $\lambda_{1}(u, v)$ is the union of two nonparallel rays from the origin. The level curves of $\lambda_{1}$ are shown in Figure 12. When $a=3 b^{2} / 4$, the level curves of $\lambda_{1}$ are parabolas, while for $a<3 b^{2} / 4$ the level curves are ellipses. It is easy to verify the convexity of $K_{c}$ in each case. 


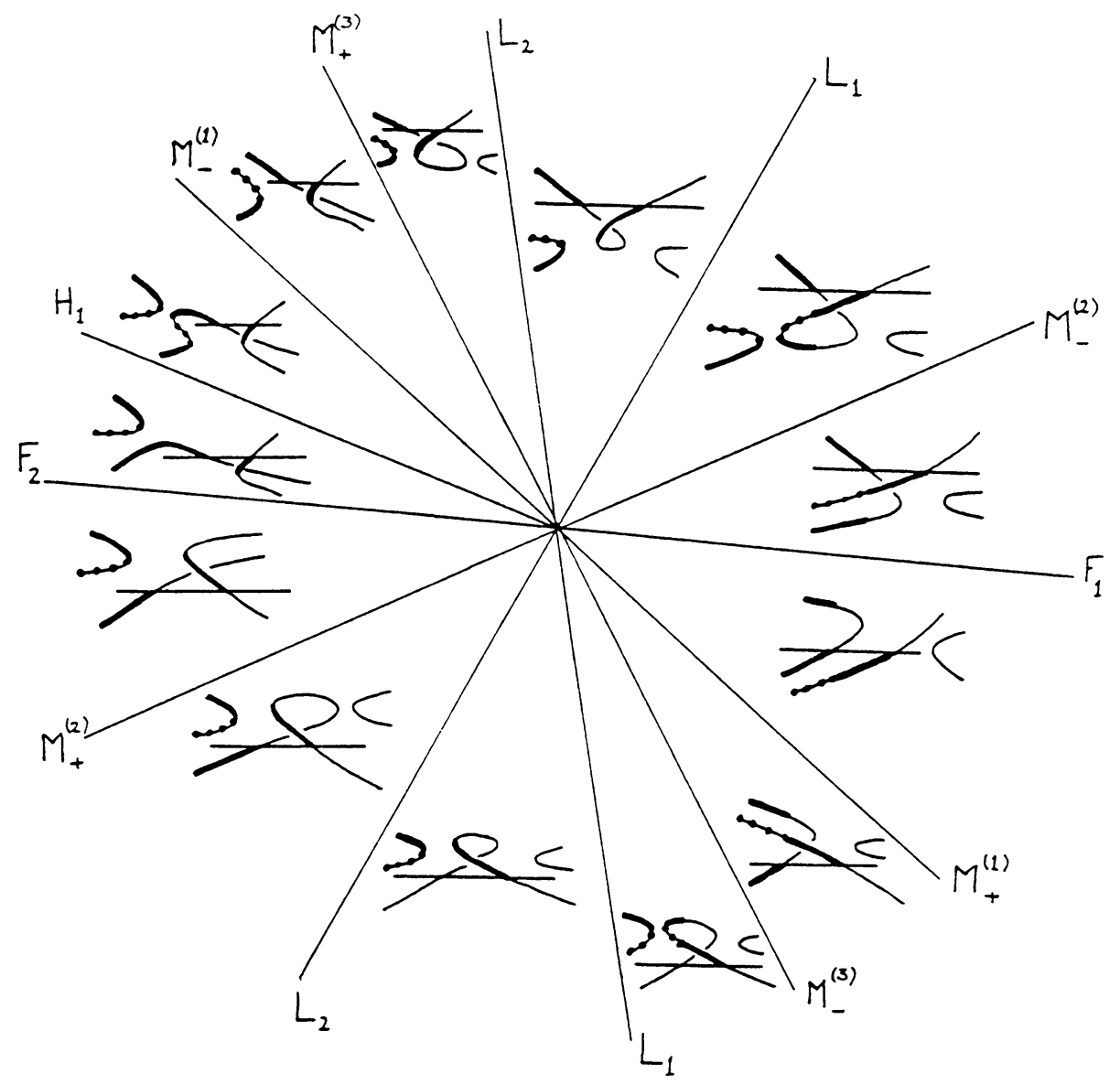

FIGURE 14. Bifurcation diagrams, Case III

LEMMA 5.3. There are no shocks satisfying (5.1).

PRoof. Let $U_{L}, U_{R}$ be joined by a shock with speed $s=s\left(U_{L}, U_{R}\right)$ satisfying (5.1). Let $c=\lambda_{1}\left(U_{R}\right)$ and consider the convex set $K_{c}$ of Lemma 5.2. By (5.1), we have $U_{L} \in K_{c}$, so that $\left(U_{L}+U_{R}\right) / 2 \in K_{c}$, by convexity. Thus

$$
\lambda_{1}\left(\left(U_{L}+U_{R}\right) / 2\right) \geqslant \lambda_{1}\left(U_{R}\right) .
$$

Now Lemma 4.3 guarantees that

$$
s=\lambda_{k}\left(\left(U_{I}+U_{R}\right) / 2\right) \text { for } k=1 \text { or } 2 .
$$

If $k=1$ in (5.4), then (5.3) and (5.4) together contradict (5.1) unless $\lambda_{1}\left(U_{L}\right)=$ $\lambda_{1}\left(U_{R}\right)=0$, since $U_{L}, U_{R}$ and $\left(U_{L}+U_{R}\right) / 2$ all lie in $K_{c}$. Alternatively, if $k=2$ in (5.4), then

$$
s=\lambda_{1}\left(\left(U_{I}+U_{R}\right) / 2\right) \geqslant \lambda_{1}\left(\left(U_{L}+U_{R}\right) / 2\right) .
$$




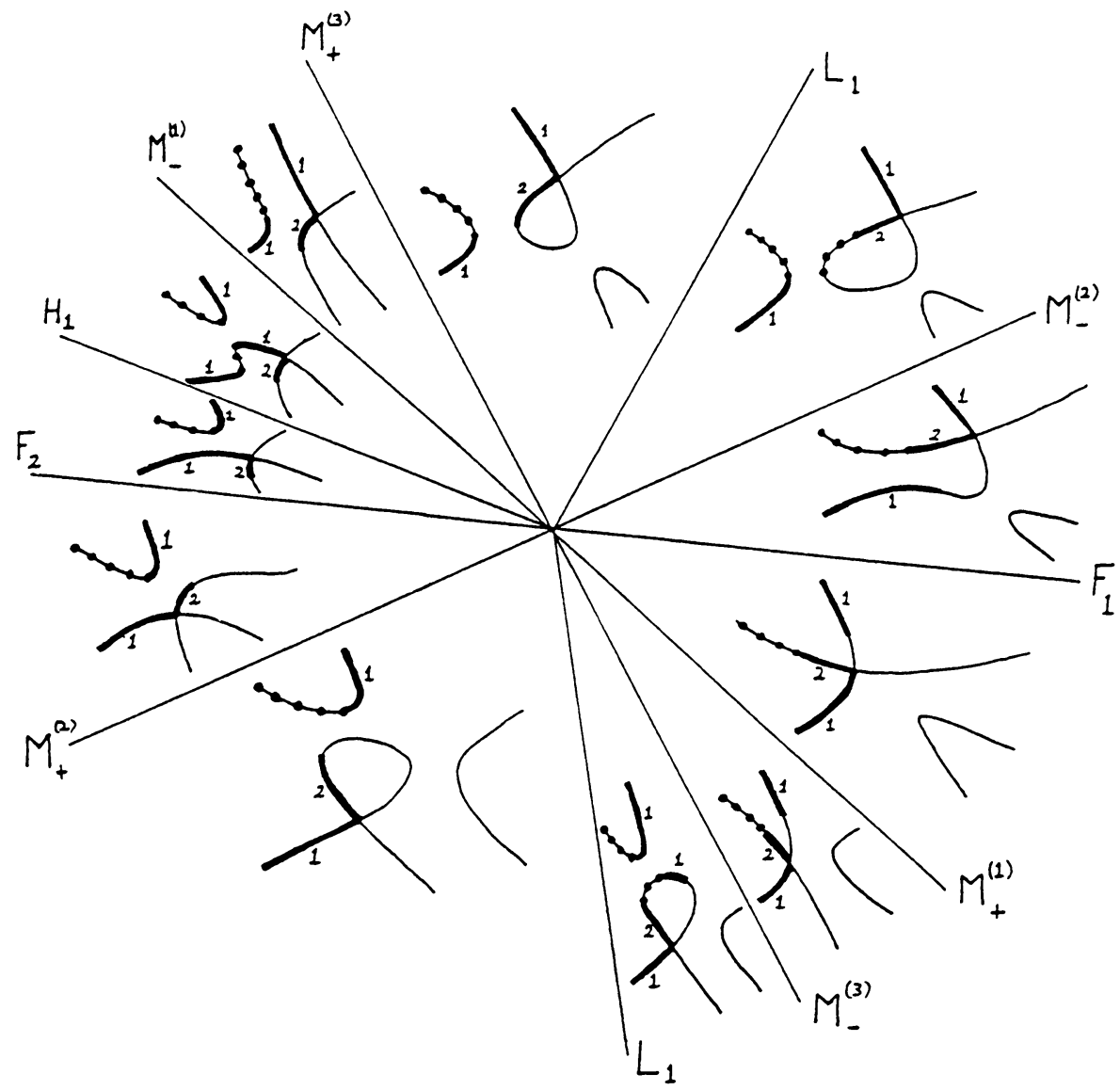

FIGURE 15. Hugoniot loci, Case III

However, (5.1), (5.3) imply

$$
s \leqslant \lambda_{1}\left(\left(U_{L}+U_{R}\right) / 2\right) .
$$

Inequalities (5.5), (5.6) give $s=\lambda_{1}\left(\left(U_{L}+U_{R}\right) / 2\right)$, which returns us to $k=1$ in (5.4).

Regarding the case $\lambda_{1}\left(U_{L}\right)=\lambda_{1}\left(U_{R}\right)=0$, we have $\lambda_{2}\left(\left(U_{L}+U_{R}\right) / 2\right)>0$, by the convexity of $K_{0}$. Thus, we cannot have $k=2$ in (5.4), since $s=0$, from (5.1). Finally, we cannot have $k=1$ in (5.4) because if $s=\lambda_{1}\left(\left(U_{L}+U_{R}\right) / 2\right)=\lambda_{1}\left(U_{R}\right)$, then $U_{L}, U_{R}$ and $\left(U_{L}+U_{R}\right) / 2$ all lie on the same straight line segment of $\lambda_{1}(U)=0$. This is not parallel to any 1-rarefaction curve, as can be seen by comparing Figures 2 and 12. The proof of Lemma 5.3 is complete.

The corresponding result for 2 -shocks is

LEMMA 5.4. There are no shocks satisfying $s=\lambda_{2}\left(U_{L}\right) \geqslant \lambda_{2}\left(U_{R}\right)$.

An immediate consequence of Lemmas 5.3, 5.4 is the following:

LEMMA 5.5. There are no shock-rarefaction 1-waves and no rarefaction-shock 2-waves. 


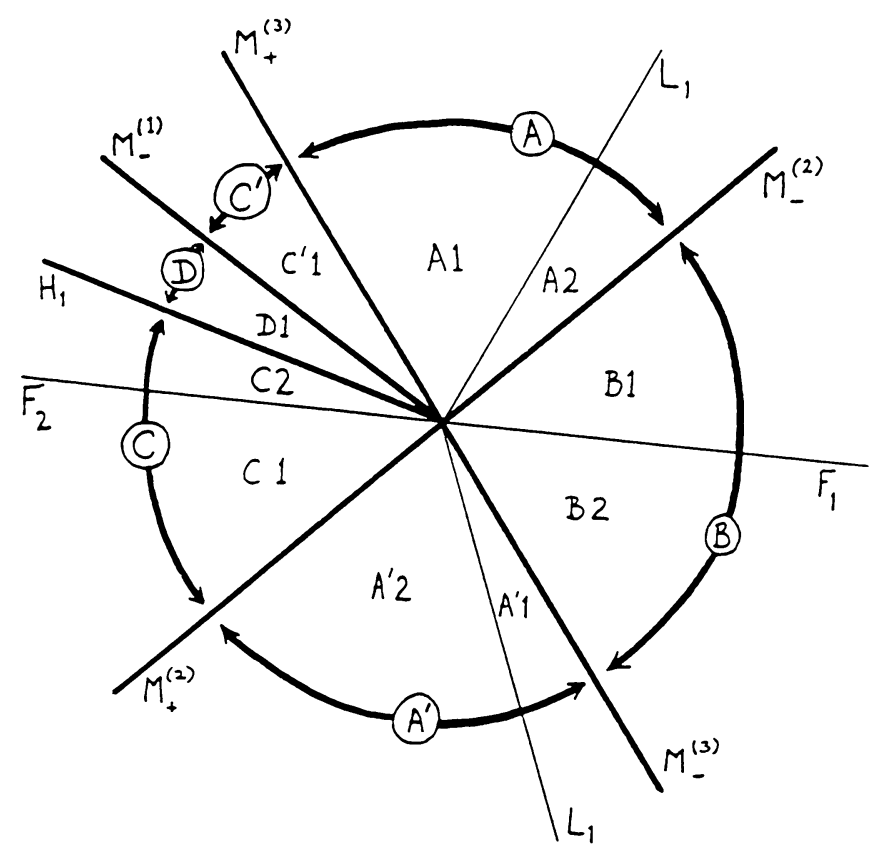

FIGURE 16. $U_{I}$-boundaries, Case III

As mentioned above, Corollary 5.5 rules out $R S R$ and $S R S$ composite waves, thus completing the proof of Theorem 5.1.

In this paper, we have found it appropriate to use the Lax admissibility condition for shock waves. However, a closer look at shock admissibility criteria for equations with an umbilic point reveals several unresolved issues. We conclude this section by discussing these issues.

Recall the generalization to $2 \times 2$ systems, due to Liu [11] of Oleinik's admissibility condition (E) for shocks. This concerns $U_{L}, U_{R}, s$ that satisfy the RankineHugoniot condition (2.2), with $U_{L}, U_{R}$ on the same curve in the Hugoniot locus $H\left(U_{L}\right)$. If $U \in H\left(U_{L}\right)$, let $s\left(U, U_{L}\right)$ be the corresponding shock speed. Then the shock wave $U(x, t)$ given by (2.3) satisfies condition (E) if

$$
s\left(U, U_{L}\right) \geqslant s\left(U_{R}, U_{L}\right)
$$

for all $U \in H\left(U_{L}\right)$ between $U_{I}$ and $U_{R}$.

For conservation laws that need not be genuinely nonlinear, the Riemann problem can have multiple solutions, all of whose shocks satisfy the Lax condition. Condition (E) overcomes this nonunqueness problem in many cases. Since genuine nonlinearity fails in any neighborhood of an umbilic point, one might expect that some form of condition (E) would be needed to guarantee uniqueness of solutions of the Riemann problem near an umbilic point. The following three points emphasize how misleading this expectation is, in the present context. First, the Lax condition on shocks 


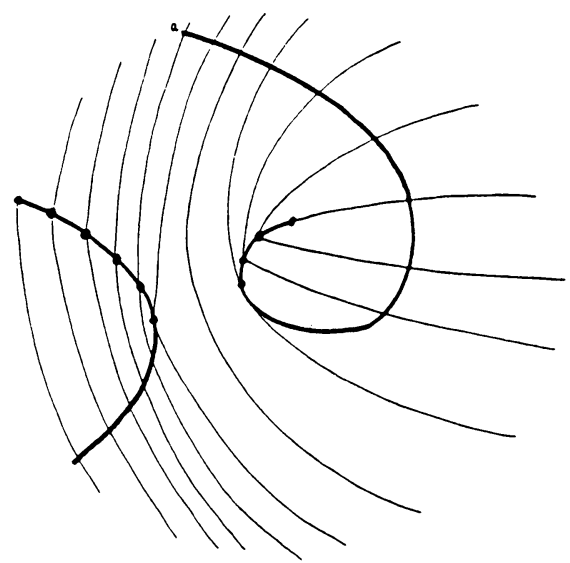

A

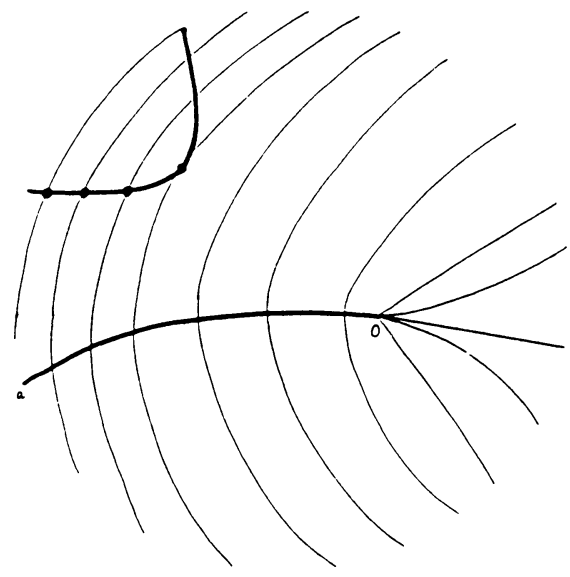

C

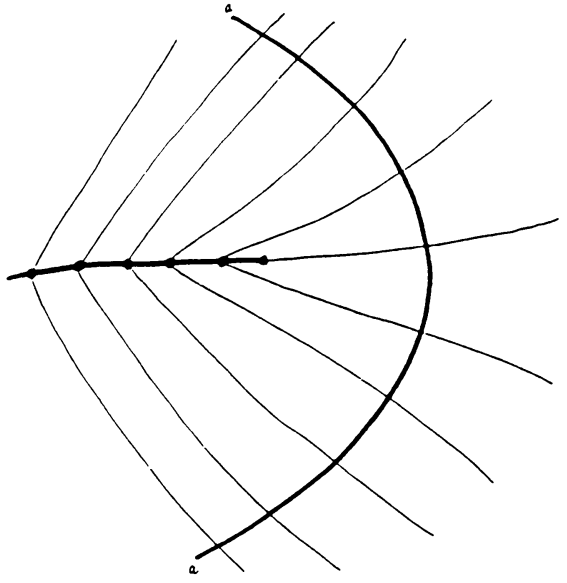

$B$

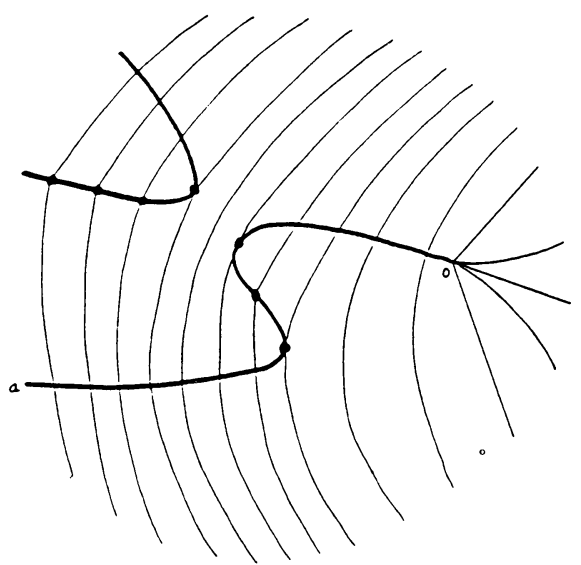

D

FIGURE 17. $U_{R}$ wave curves, Case III

gives uniqueness of solutions of the Riemann problem. Second, some of the Lax shocks used to construct the solution in Cases II-IV fail to satisfy condition (E). Thus, the use of condition (E) would mean the loss of existence of solutions for some data. Finally, in Case I, not even the Lax condition is inclusive enough to give existence of solutions for all data; there appear to be physical undercompressive shocks, not satisfying the Lax condition, that are needed to solve the Riemann problem. (Cf. [6, 14].)

To expand upon the first and second of these observations, note that nonuniqueness for a single conservation law, and indeed for the strictly hyperbolic systems considered by Liu [11], arises because of $R S R$ and $S R S$ composite waves. Theorem 5.1 shows that these composite waves are not present for equations (1.3) with 


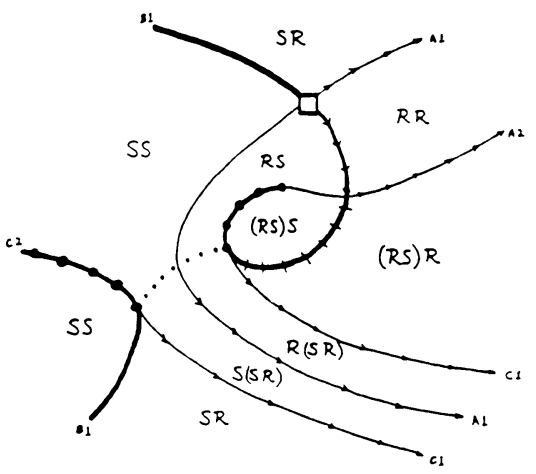

(A1)

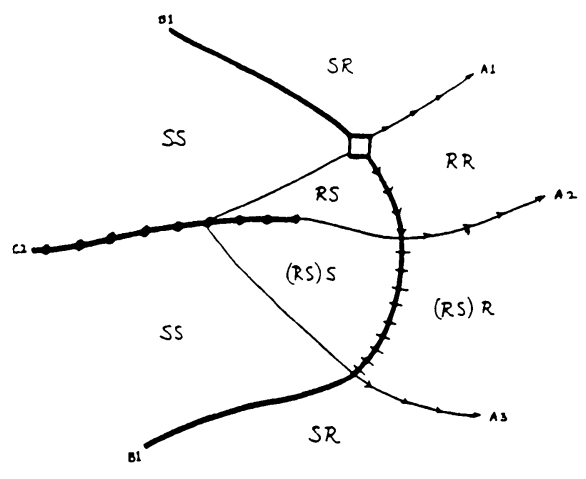

(B1)

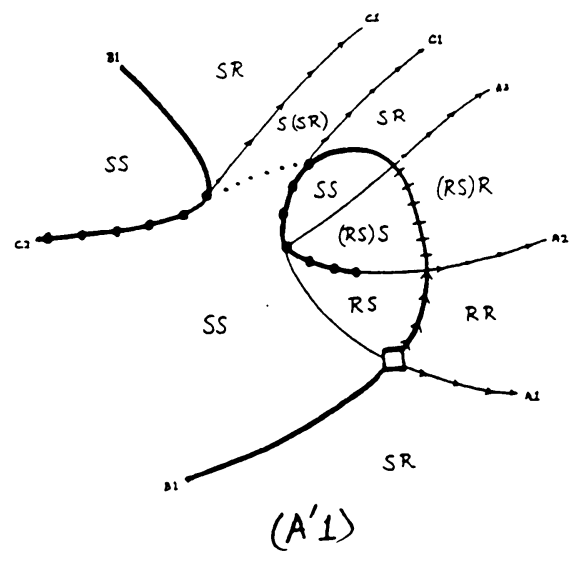

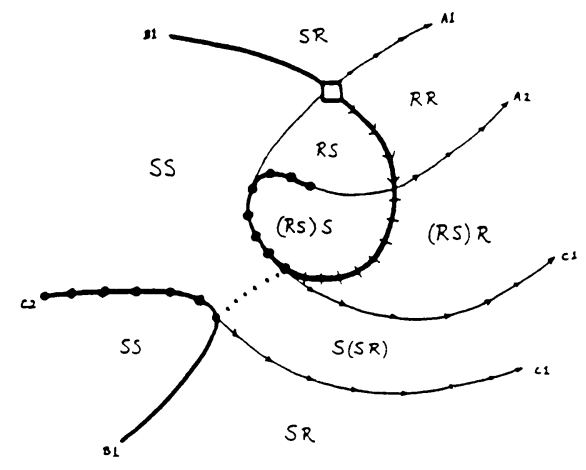

(A2)

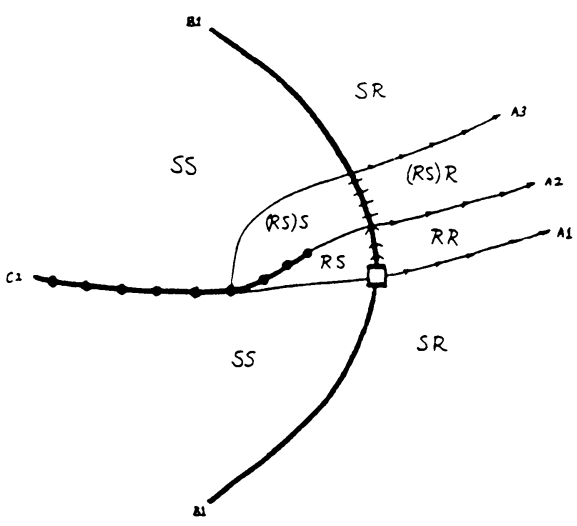

(B2)

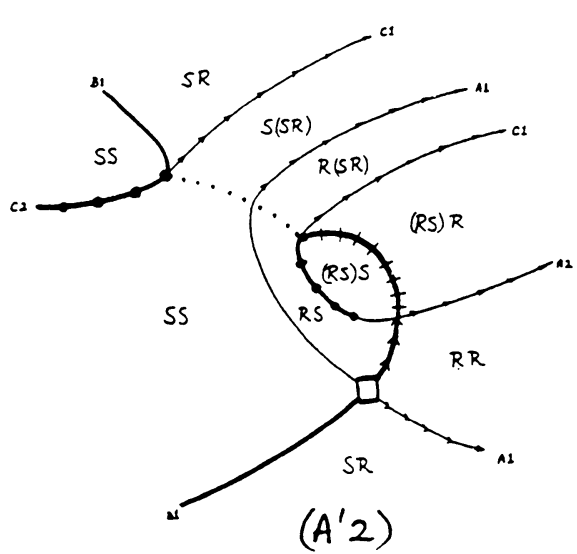

FIGURE 18. $U_{R}$-regions, Case III 


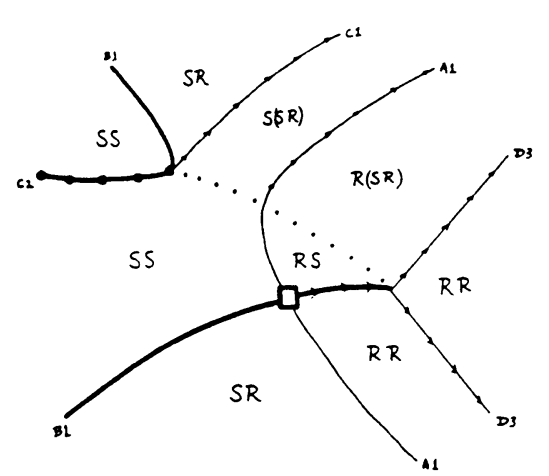

(C1)

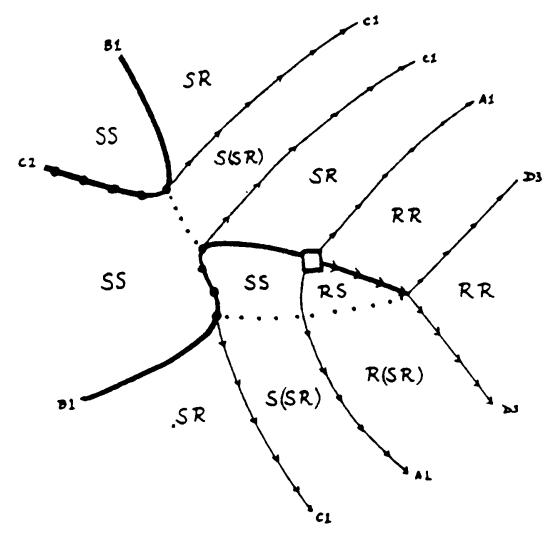

(D1)

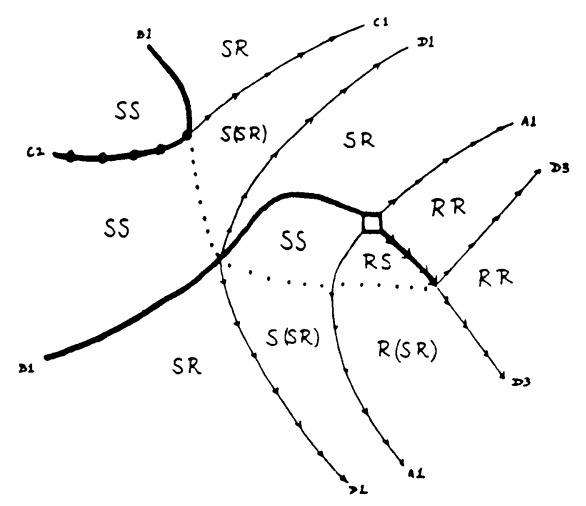

(c2)

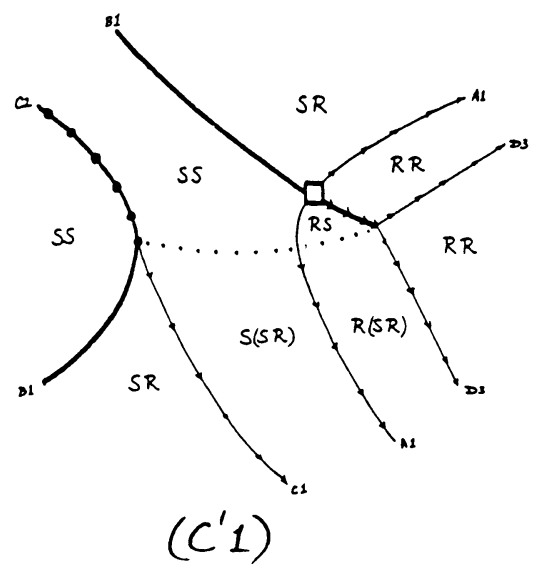

FIGURE 18. $U_{R}$-regions, Case III (continued)

quadratic nonlinearities, despite the loss of genuine nonlinearity. Some understanding of this point may be achieved by considering a single conservation law

$$
u_{t}+f(u)_{x}=0 .
$$

Genuine nonlinearity for (5.8) corresponds to the convexity condition

$$
f^{\prime \prime}(u) \neq 0 .
$$

If the flux function $f$ has a single inflection point, then the Lax condition and the Oleinik condition (E) are equivalent. In fact, there are no $R S R$ or $S R S$ composite 


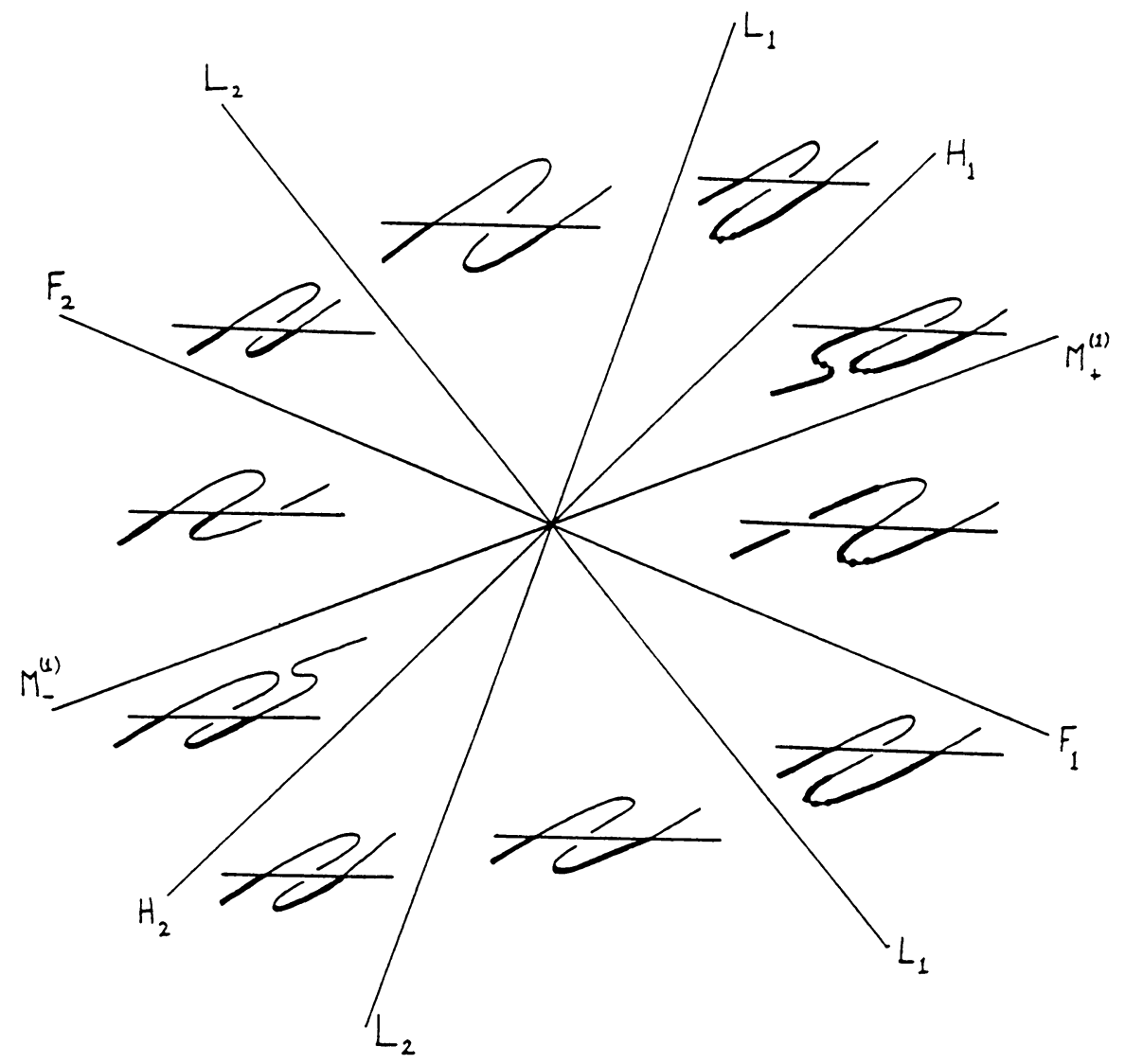

Figure 19. Bifurcation diagrams, Case IV

waves; the only composite waves are $R S$ (if the characteristic speed $f^{\prime}(u)$ has a maximum), or $S R$ (if $f^{\prime}(u)$ has a minimum). Note that the Buckley-Leverett equation of two-phase flow in a porous medium is an example of equation (5.8) in which the corresponding flux function $f$ has a single inflection point.

If $f$ has two inflection points, then $S R S$ or $R S R$ constructions are possible, and the Lax condition is insufficient to guarantee uniqueness. In Figure 13, we show the familiar example of two solutions of a Riemann problem, whose shocks satisfy the Lax condition. Condition (E) is satisfied only by the solution having composite waves.

In the second observation above, we remarked that some of the Lax shocks used in this paper to construct the solution of the Riemann problem fail to satisfy condition (E). As an example, in Case II, consider $\left(s, U_{R}\right)$ given by point $P$ in Figure 4. This point clearly fails to satisfy condition $(E)$. A subtle feature of this example is that condition (E) fails because $s\left(U, U_{L}\right)=\lambda_{2}(U)$ at a point $U$ between $U_{L}$ and $U_{R}$ in $H\left(U_{I}\right)$. That is, monotonicity of $s$ along the Hugonioit locus from $U_{I}$ to $U_{R}$ fails 


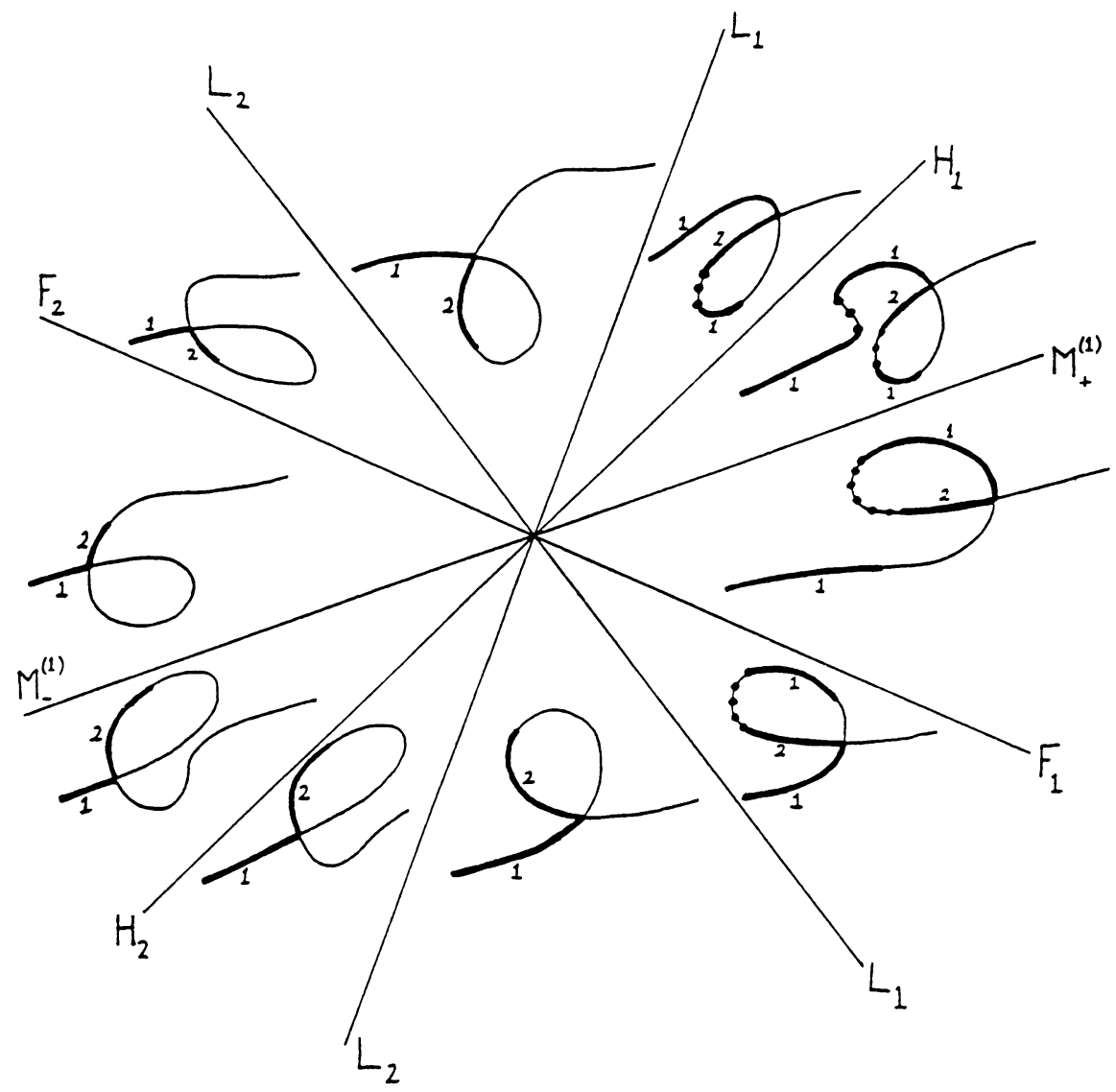

Figure 20. Hugoniot loci, Case IV

due to the opposite characteristic family (here, the 2-waves) from that of the shock (here, a 1-shock).

Although we have shown that condition (E) is too restrictive near an umbilic point, we should emphasize that some form of condition (E) will be required when considering strong shocks in the full model of three-phase flow (see [13]), including cubic and higher order terms. In fact, nonuniqueness of Lax admissible solutions, in a single characteristic family, does arise in this context.

However, there are two immediate difficulties which must be overcome to apply condition (E) to equations with umbilic points. The first is that the portion of $H\left(U_{L}\right)$ between $U_{L}$ and $U_{R}$ is not uniquely defined when $H\left(U_{L}\right)$ has a loop, as in Figures 15, 20. This difficulty is only apparent, however-if we work with the bifurcation diagram rather than its projection $H\left(U_{L}\right)$ onto the $U_{R}$-plane, then the ordering of $U$ in (5.7) becomes unambiguous. A more serious difficulty is that the Hugoniot locus is often disconnected near an umbilic point, and there is no analogue of condition (E) for values of $U_{R}$ on a detached portion of $H\left(U_{L}\right)$. 


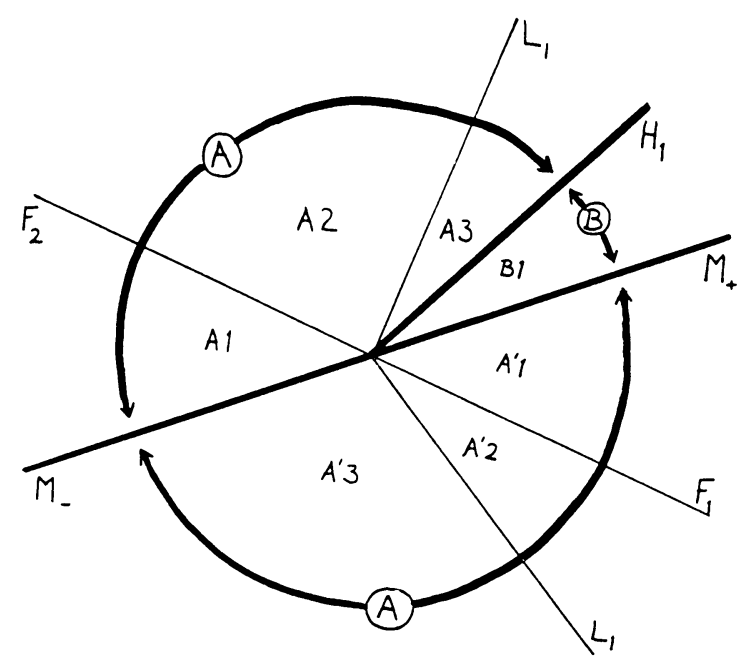

FIGURE 21. $U_{I}$-boundaries, Case IV

Appendix 1: Corners of $U_{R}$-regions. In this Appendix we consider intersections of $U_{R}$-boundaries, i.e., corners of the $U_{R}$-regions. Specifically, we compile a list of intersection points that arise for quadratic nonlinearities in Cases II, III, or IV. This information may help one understand how the $U_{R}$-regions fit together.

An intersection point results when two or more of the boundary curves classified in Table 1 meet one another. Thus to each such intersection point $\hat{U}$ may be associated a subset of entries of Table 1; viz., the types of boundary curves which touch $\hat{U}$. The simplest intersection points are those associated to a pair of entries of Table 1, one coming from column A and one from column B. At such intersection points two boundary curves of a type familiar from strictly hyperbolic equations cross one another, resulting in a corner shared by four regions. For example, $U_{L}$ itself is of this type. We ignore this simple class of intersection points and consider only the others.

In Table 2 we have listed the seven types of intersection points, exclusive of the simple cases just mentioned. The points, which are listed by the corresponding subset of entries of Table 1, are grouped by which pair of columns of Table 1 are represented in the subset-it turns out that in each case exactly two columns are represented. (Remark: Of the six possible pairs of columns, only three appear in Table 2. $\mathrm{AB}$ was dismissed above, and neither $\mathrm{AD}$ nor $\mathrm{CD}$ occurs.) For each intersection point, the underlying phenomenon is briefly described, and one figure containing a point of the type in question is referenced. Our description of the phenomenon is somewhat arbitrary in that we have selected just one of several interrelated phenomena occurring at each point. For example, we describe A1, A3, $(\mathrm{C} 2)_{\mathrm{i}},(\mathrm{C} 2)_{\mathrm{ii}}$ by " $(R S)_{0}$ changes to $S_{0}$ ", but we could have equally well described such points by "A1 and A3 boundaries (which are 2-shock curves) collide". Useful insight can be gained by exploring these interrelationships, but we do not pursue this here. 
TABLE 2. Listing of intersection points

\begin{tabular}{cccc}
$\begin{array}{c}\text { Pair of } \\
\text { columns }\end{array}$ & $\begin{array}{c}\text { Subset of } \\
\text { entries }\end{array}$ & Phenomenon & Reference figure \\
\hline $\mathrm{AC}$ & $\mathrm{A} 2,(\mathrm{C} 2)_{\mathrm{ii}}$ & $\begin{array}{c}\text { End of }(R S)_{0} \\
(R S)_{0} \text { changes to } S_{0}\end{array}$ & $10 . \mathrm{C} 1$ \\
& $\begin{array}{c}\mathrm{A} 1, \mathrm{~A} 3,(\mathrm{C} 2)_{\mathrm{i}} \\
(\mathrm{C} 2)_{\mathrm{ii}}\end{array}$ & $10 . \mathrm{A} 1$ \\
\hline $\mathrm{BC}$ & $\mathrm{B} 1, \mathrm{~B} 2,(\mathrm{C} 1)_{k}$, & $W_{1}\left(U_{L}\right)$ changes to $S_{0}$ & $10 . \mathrm{A} 1$ \\
$(\mathrm{C} 2)_{k}$ & & \\
& $(k=\mathrm{i}$ or ii) & & $10 . \mathrm{A} 1$ \\
$\mathrm{BD}$ & $\mathrm{B} 2,(\mathrm{D} 2)_{\mathrm{i}}$ & $\begin{array}{c}\text { Secondary bifurcation } \\
\left(\text { at } U^{* *}\right)\end{array}$ \\
& $\mathrm{B} 2,(\mathrm{D} 2)_{\mathrm{ii}}$ & $\begin{array}{c}\text { Start of detached } \\
\text { rarefaction curve }\end{array}$ & $10 . \mathrm{C} 1$ \\
& $\mathrm{~W} 1, \mathrm{~B} 2, \mathrm{D} 1$ & $W_{1}\left(U_{L}\right)$ crosses $F_{2}$ & $10 . \mathrm{A}^{\prime} 1$ \\
& $\mathrm{~B} 1, \mathrm{~B} 2, \mathrm{D} 3$ & $W_{1}\left(U_{L}\right)$ stops at origin \\
& & $($ Case III only $)$ & $18 . \mathrm{C} 1$ \\
& & &
\end{tabular}
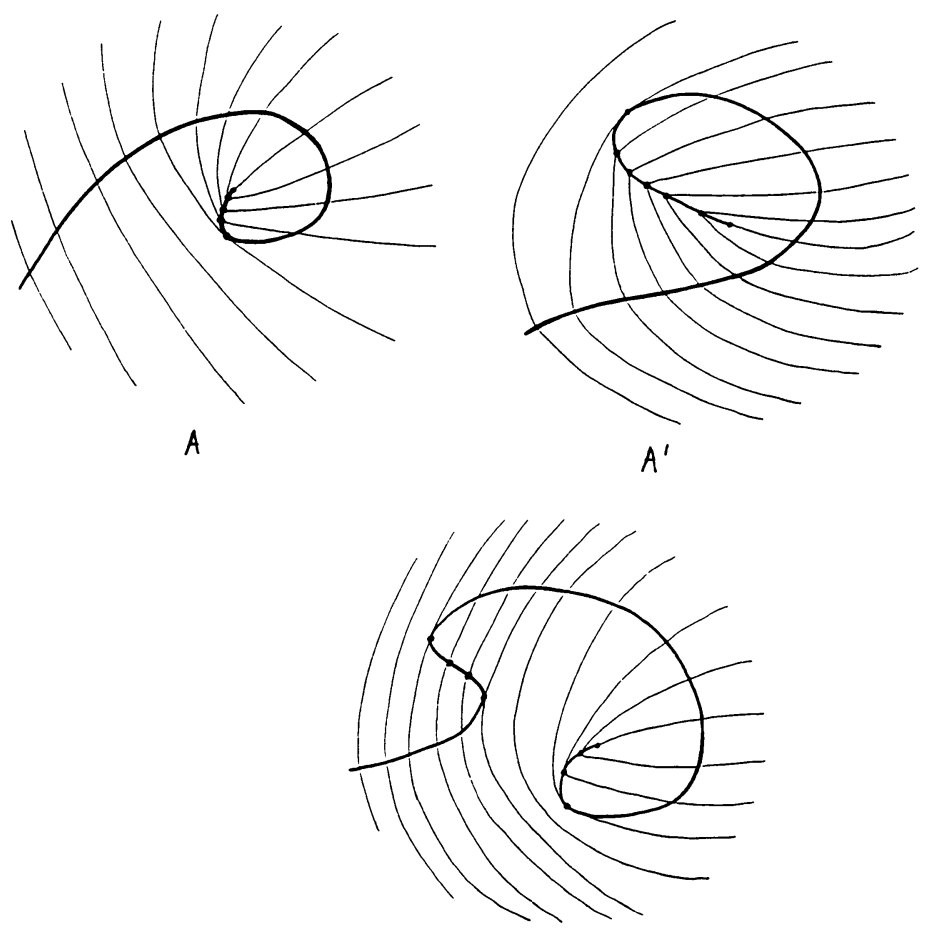

FIGURE 22. $U_{R}$ wave curves, Case IV 


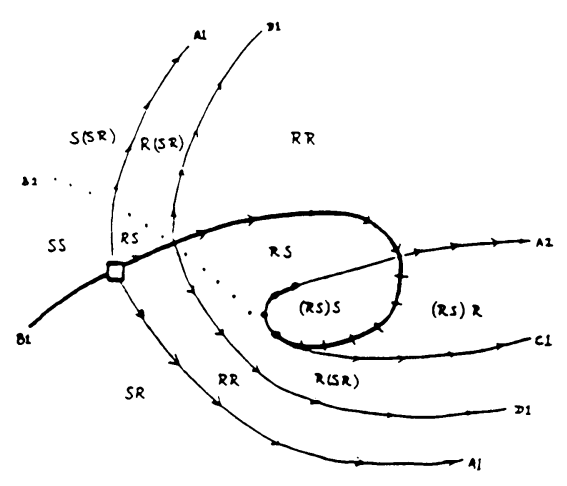

(A1)

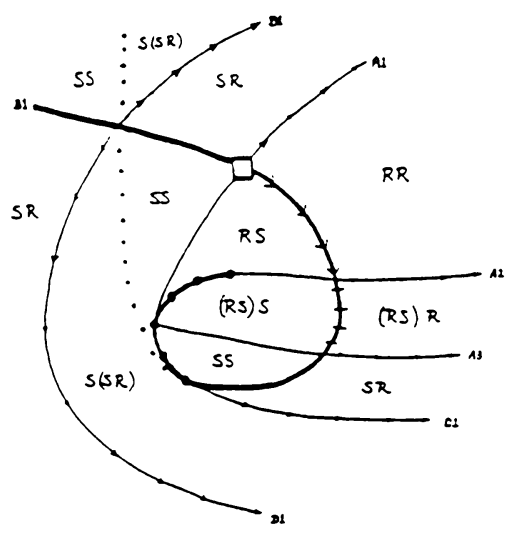

(A3)

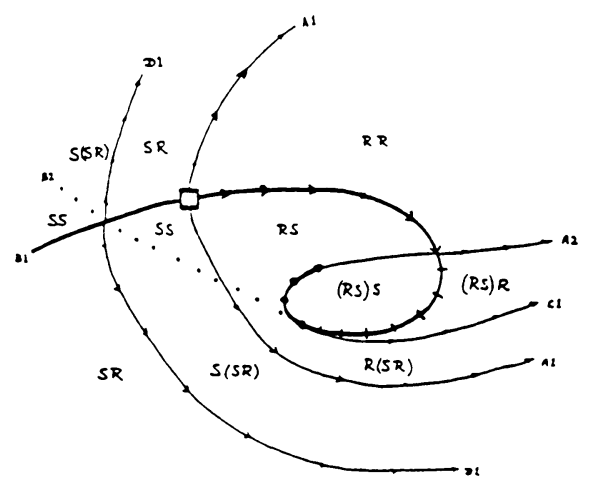

(A2)

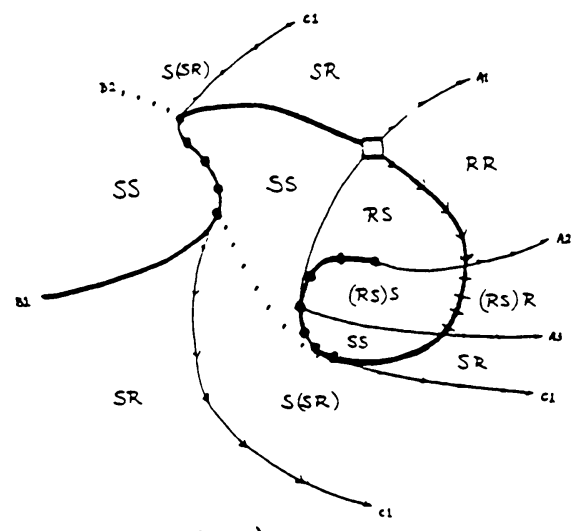

(B1)

FIGURE 23. $U_{R}$-regions, Case IV

Appendix 2: Cases III and IV. This appendix was prepared by Eli Isaacson ${ }^{1}$ (Department of Mathematics, University of Wyoming, Laramie, Wyoming 82701). Dan Marchesin² (Department of Mathematics, PUC-RJ, 22453 Rio de Janeiro, Brazil), David G. Schaeffer, and Michael Shearer.

In it, we give the solution of the Riemann problem in Cases III and IV. As for Case II, the solution is deduced from the pattern of rarefaction curves (Figure 2), and the bifurcation diagrams/Hugoniot loci given in Figures 14-15, 19-20. In Figures 16, 21, we show the $U_{L}$-plane with major and minor boundaries. The wave curves are shown in Figures 17, 22 for each $U_{L}$-sector, and the $U_{R}$-regions are displayed in Figures 18, 23.

\footnotetext{
${ }^{1}$ Supported in part by FINEP/Brazil Grant 4.3.82.017.9, CNPq/Brazil Grant 1.01.10.011/84-ACI, and NSF Grant 5274298 INT-8415209.

${ }^{2}$ Supported in part by FINEP/Brazil Grant 4.3.82.017.9, CNPq/Brazil Fellowship 30.0204/83-MA, $\mathrm{CNPq} / \mathrm{Brazil}$ Grant 1.01.10.011/84-ACI, and NSF Grant 5274298 INT-8415209.
} 


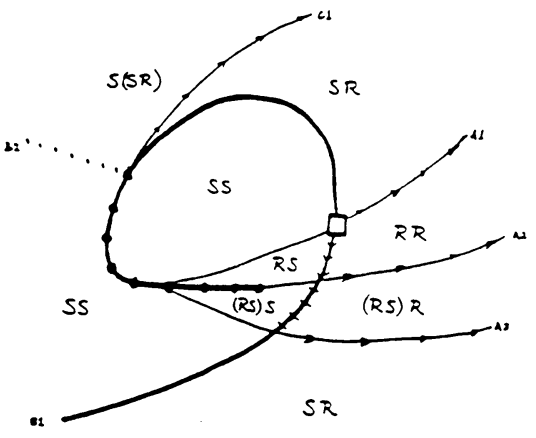

(A'1)

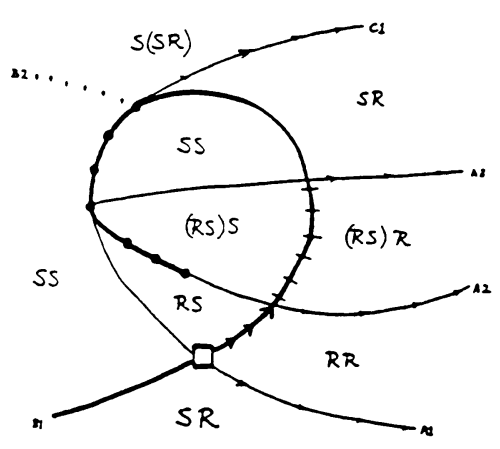

( $\left.A^{\prime} 2\right)$

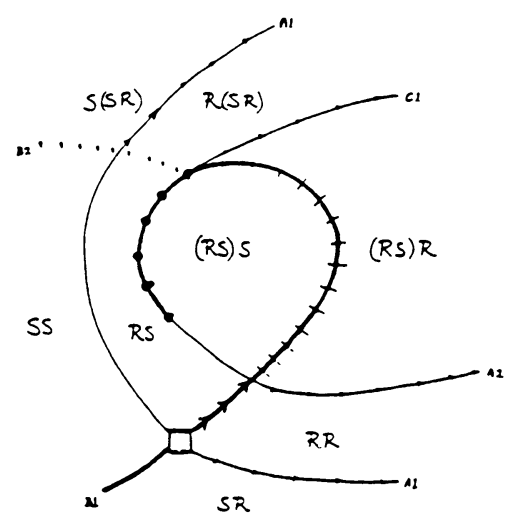

$\left(A^{\prime} 3\right)$

Figure 23. $U_{R}$-regions, Case IV (continued)

\section{REFERENCES}

1. J. B. Bell, J. A. Trangenstein and G. R. Shubin, Conservation laws of mixed type describing three-phase flow in porous media, Exxon Production Research preprint, 1985.

2. M. G. Crandall and P. H. Rabinowitz, Bifurcation from simple eigenvalues, J. Funct. Anal. 8 (1971), $321-340$.

3. J. Glimm, C. Klingenberg, O. McBryan, B. Plohr, D. Sharp and S. Yaniv, Front tracking and two dimensional Riemann problems, Adv. Appl. Math. 6 (1985), 259-290.

4. J. Glimm, E. Isaacson, D. Marchesin and O. McBryan, Front tracking for hyperbolic systems, Adv. Appl. Math. 2 (1981), 91-119.

5. M. Golubitsky and D. G. Schaeffer, Singularities and groups in bifurcation theory, Springer, New York, 1985.

6. H. Holden, On the Riemann problem for a prototype of a mixed type conservation law, Comm. Pure Appl. Math. 40 (1987), 229-264.

7. E. Isaacson, D. Marchesin, B. Plohr and B. Temple, The classification of solutions of quadratic Riemann problems. I. MRC report, Univ. of Wisconsin, 1985. 
8. E. Isaacson and B. Temple, The classification of solutions of quadratic Riemann problems. II. MRC report, Univ. of Wisconsin, 1985.

9. . The classification of solutions of quadratic Riemann problems. III, MRC Tech. Summary Report, 1986.

10. P. D. Lax, Hyperbolic systems of conservation laws. II, Comm. Pure Appl. Math. 10 (1957), 537-566.

11. T.-P. Liu, The Riemann problem for general $2 \times 2$ conservation laws, Trans. Amer. Math. Soc. 199 (1974), 89-112.

12. O. A. Oleinik, On the uniqueness of the generalized solution of Cauchy problem for nonlinear system of equations occurring in mechanics, Uspehi Mat. Nauk. (Russian Math. Surveys) 12 (1957), 169-176.

13. D. G. Schaeffer and M. Shearer, The classification of $2 \times 2$ systems of nonstrictly conservation laws. with application to oil recovery: Appendix with D. Marchesin and P. J. Paes-Leme. Comm. Pure Appl. Math. 40 (1987), 141-178.

14. M. Shearer, D. G. Schaeffer, D. Marchesin and P. J. Paes-Leme, Solution of the Riemann problem for a prototype $2 \times 2$ system of nonstrictly hyperbolic conservation laws, Arch. Rational Mech. Anal. 97 (1987), 299-320.

15. Z. Tang and T. C. T. Ting, Wave curves for the Riemann problem of plane waves in simple isotropic elastic solids, Internat. J. Engrg. Sci. (to appear).

Department of Mathematics, Duke University, Durham, North Carolina 27706

Department of Mathematics, North Carolina State University, Raleigh, North Carolina 27695 